\title{
Joint Trajectory Planning of Space Modular Reconfigurable Satellites Based on Kinematic Model
}

\author{
Jiping An $\left(\mathbb{D}\right.$, Xinhong Li $\mathbb{D}^{\mathbb{D}}$, Zhibin Zhang $\mathbb{D}$, Wanxin Man $\mathbb{D}$, and Guohui Zhang $\mathbb{D}$
}

Department of Aerospace Science and Technology, Space Engineering University, Beijing 101416, China

Correspondence should be addressed to Jiping An; 2684700589@qq.com

Received 21 July 2020; Revised 16 November 2020; Accepted 10 December 2020; Published 31 December 2020

Academic Editor: Paolo Gasbarri

Copyright ( 2020 Jiping An et al. This is an open access article distributed under the Creative Commons Attribution License, which permits unrestricted use, distribution, and reproduction in any medium, provided the original work is properly cited.

\begin{abstract}
This paper investigates the application of particle swarm optimization (PSO) algorithm to plan joint trajectories of the space modular reconfigurable satellite (SMRS). SMRS changes its configuration by joint motions to complete various space missions; its movement stability is affected by joints motions because of the dynamic coupling effect in space. To improve the movement stability in reconfiguration progress, this paper establishes the optimization object equation to characterize the movement stability of SMRS in its reconfiguration process. The velocity-level and position-level kinematic models based on the proposed virtual joint coordinate system of SMRS are derived. The virtual joint coordinate system solves the problem of asymmetric joint coordinate system resulted by the asymmetric joint arrangement of SMRS. The six-order and seven-order polynomial curves are chosen to parameterize the joint trajectories and ensure the continuous position, velocity, and acceleration of joint motions. Finally, PSO algorithm is used to optimize the trajectory parameters in two cases. Consistent optimization results in terms of the six-order and seven-order polynomial in both cases prove the PSO algorithm can be effectively used for joint trajectory planning of SMRS.
\end{abstract}

\section{Introduction}

Traditional space systems have been designed to perform a single mission, and the permanently fixed structure cannot be reconfigured for other missions. Furthermore, long development cycle and the launch window limitation lead to the difficulties of current space system to respond quickly to emergencies [1]. To address the limitations of existing space systems, future space systems are expected to have multiple uses and react to unforeseen events [2]. Under this goal, some new spacecraft concepts are proposed, such as cubic cellular satellites [3], satellite formations [4], and reconfigurable satellites [1]. Reconfigurable satellite is a kind of space system with configuration adaptability. It carries multiple types of task subsystem whose space position can be arranged and reorganized by structural reconfiguration to have the ability to perform multiple space missions. For example, one reconfigurable satellite carrying multiple optical cameras could change the space directions of the cameras through the reconfigurable mechanism and splice the fields of view of all cameras to expand the imaging area or, respectively, reconnoiter different targets.

The space modular reconfigurable satellite (SMRS) proposed in this paper is a new type of reconfigurable satellite, which is born with the diversity of space mission but the single mission function of current satellite systems. SMRS is a chain structure satellite composed of different functional modules and joints between the modules (see Figure 1) and could change its configuration adaptively according to the adjustment of space mission. Each module of SMRS is the integration of satellite payloads or subsystems with standard interface and regular shape. The modular design is convenient for the folding and package of satellite in rocket and also for the reconfiguration in space. Different from the reconfigurable satellites that used own manipulators [5], external service robots or autonomous flight [6] to change their configuration, SMRS change its configuration by joint motions. Each module of SMRS is connected by three 


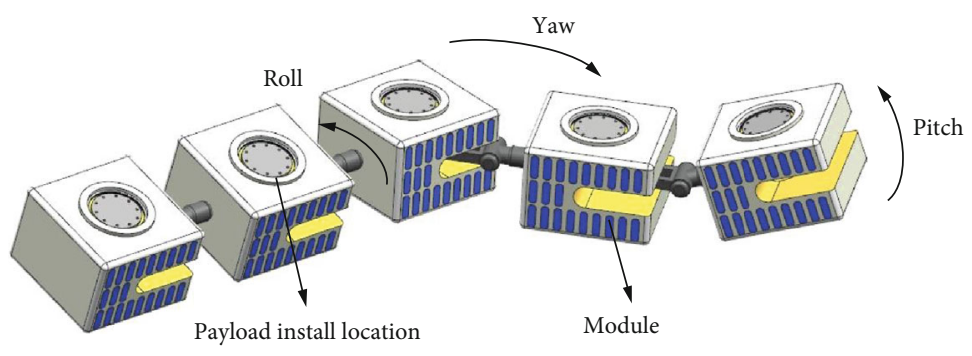

FIGURE 1: Model of SMRS.

rotation joints that are mutually orthogonal and provide the freedom degrees of pitch, roll, and yaw between modules. Different configurations are realized by relative rotation between modules. Thus, SMRS can adjust its configuration adaptively according to the requirements of space mission and the mission capability of SMRS is improved accordingly. The structure of 5-module SMRS is shown in Figure 1. What should be noted is that the type and number of satellite modules and payloads carried can be configured according to the requirements of space missions.

SMRS requires multiple reconfigurations in space to adapt to the various space missions. Unlike the reconfigurable system with fixed base on the ground, SMRS is with such a characteristic that any joint motion will affect the pose of other modules because of the floating rootless state of the space system, which is also known as the dynamic coupling effect between joint motion and pose of modules [7, 8]. The dynamic coupling effect can be described by the positionlevel and velocity-level kinematic model of SMRS.

The dynamic coupling effect makes the changes in pose of space reconfigurable system more complicated. In order to reduce the impact of the dynamic coupling effect or use it, the joint trajectory of space reconfigurable system such as space robots is planned by many researchers. Under different space mission requirements, the forms of optimization objective for joint trajectory planning are not uniform. In space missions, common optimization objective includes the following: (1) the minimum disturbance of the base [911]. When a space system carries numerous precision instruments and payloads with high requirements for orientation in the base, the base disturbance is required to be small in the reconfiguration process; (2) minimum pose error of end effector. For the space reconfigurable missions, such as space capture, which requires high precision of the final pose of the end effector, the error between the end effector and its desired pose should be reduced as much as possible; (3) minimum energy consumption [12]; (4) optimal reconfigurable time $[13,14]$, for temporary or more urgent space missions; (5) obstacle avoidance [12]; (6) the lowest jerk [15]; and (7) minimum joint rotation angle [14].

However, a special characteristic for SMRS is that it may carry payloads in every module. To maintain their continuous working state, severe pose changes are not allowed in the reconfiguration process. For example, severe pose disturbance may cause communication interruption between satellite and ground control center [10]. At the same time, as a multijoint chain system, severe pose change will lead to sys- tem vibration and other dynamic problems. Hence, this paper explores the feasibility of using trajectory planning method to improve the stability of SMRS and puts forward the procedures and methods in detail.

The logic of joint trajectory planning of SMRS is to set the optimization objective equation and define the control parameters of the joint trajectory which are the typical parameters that can define the joint trajectory. According to the optimization objective equation, the relationship between the control parameters and the optimization objective equation is derived. Under a series of equality or inequality constraints, the optimization algorithm is used to iteratively optimize the control parameters, and then optimized control parameters are used to obtain a specific joint trajectory. Therefore, the complete joint trajectory planning process mainly includes the following steps: (1) establish an optimization objective equation, (2) parameterize joint trajectory and define control parameters, (3) select an optimization algorithm to optimize the control parameters, and (4) solve the optimized joint trajectory.

The first problem encountered is not only the characterization of reconfiguration stability of SMRS but also the form of optimization objective equation. Because of the equal importance of each module of SMRS, feasible optimization objective equation can be weighted sum of each module pose velocity. However, this form of the optimization objective equation will be too complicated to impose high requirements on space computing resources. Therefore, this paper derives the relationship between the module pose and the joint trajectory to propose a more simplified and practicable optimization objective equation and clarifies the application of the PSO algorithm for joint trajectory planning under multiple joint constraints.

Based on this, the paper is arranged as follows: in Section 2 , we establish the velocity-level and position-level kinematic equations of the SMRS to describe the dynamic coupling effect between pose velocity and joint trajectories; in Section 3 , the optimization objective equation and constraints of joint trajectory planning are determined, and the joint trajectory is parameterized to determine the optimal parameters of the joint trajectory; in Section 4, we analyze the principle and steps of the PSO algorithm used in joint trajectory planning of SMRS; In Section 5, the PSO algorithm is used to plan the trajectories of SMRS from folding configuration to deployment configuration and from deployment configuration to mission configuration; in Section 6, we discuss and summarize the work. 
TABLE 1: Kinematic and dynamic symbols of SRMS.

\begin{tabular}{|c|c|}
\hline Symbols & Representation \\
\hline$\sum_{I}$ & The inertia coordinate system \\
\hline$\sum_{b}$ & Base coordinate system \\
\hline$\sum_{0}$ & The center of mass coordinate system of $B_{0}$ \\
\hline$\sum_{i}^{k}$ & The center of mass coordinate system of $B_{i}^{k}$ \\
\hline $\mathrm{CM}$ & Center of mass of SMRS \\
\hline$C_{i}^{k},(k=a, b)$ & The center of mass of $B_{i}^{k}$ \\
\hline $\mathbf{a}_{i}^{\mathbf{k}} \in \mathbf{R}^{3},(k=a, b)$ & Position vectors from $J_{i}^{k}$ to $C_{i}^{k}$ \\
\hline $\mathbf{b}_{\mathbf{i}}^{\mathbf{k}} \in \mathbf{R}^{3},(k=a, b)$ & Position vectors from $C_{i}^{k}$ to $J_{i+1}^{k}$ \\
\hline $\mathbf{r}_{\mathbf{i}}^{\mathbf{k}} \in \mathbf{R}^{3},(k=a, b)$ & The position vector of $C_{i}^{k}$ based on $\sum_{I}$ \\
\hline $\mathbf{p}_{\mathbf{i}}^{\mathbf{k}} \in \mathbf{R}^{3},(k=a, b)$ & The position vector of $J_{i}^{k}$ based on $\sum_{I}$ \\
\hline $\mathbf{k}_{\mathbf{i}}^{\mathbf{k}} \in \mathbf{R}^{3},(k=a, b)$ & The unit vector representing the rotation direction of $J_{i}^{k}$ \\
\hline$\dot{\mathbf{x}}_{\mathbf{i}}^{\mathbf{k}} \in \mathbf{R}^{6},(k=a, b)$ & The linear velocity and angular velocity of $B_{i}^{k},(k=a, b)$ \\
\hline$\dot{\mathbf{x}}_{0} \in \mathbf{R}^{6}$ & The linear velocity and angular velocity of $B_{0}$ \\
\hline$\Theta^{\mathbf{k}} \in \mathbf{R}^{n_{k}},(k=a, b)$ & The actual joint angle vector of arm- $k$ \\
\hline$\Theta_{\mathrm{d}}^{\mathbf{k}} \in \mathbf{R}^{n_{k}},(k=a, b)$ & The desired joint angle vector of arm- $k$ \\
\hline$m_{0}$ & The mass of $B_{0}$ \\
\hline$m_{\mathbf{i}}^{\mathbf{k}},(k=a, b)$ & The mass of $B_{i}^{k}$ \\
\hline$M$ & Total mass of the system \\
\hline $\mathbf{I}_{0} \in \mathbf{R}^{3 \times 3}$ & The inertia matrices of $B_{0}$ referred to $\sum_{0}$ \\
\hline $\mathbf{I}_{\mathbf{i}}^{\mathbf{k}} \in \mathbf{R}^{3 \times 3}$ & The inertia matrices of $B_{\mathrm{i}}^{k}$ referred to $\sum_{i}^{k}$ \\
\hline $\mathbf{E}_{3}$ & $3 \times 3$ identity matrix \\
\hline$\theta_{i}^{k}$ & The angle of $J_{i}^{k}$ \\
\hline
\end{tabular}

\section{Kinematic Model of SMRS}

Due to the dynamic coupling effect between the pose of each module and the joint trajectories, the trajectory planning in space is more complex than that of reconfigurable mechanism on fixed base [16]. When planning the trajectory of each joint to improve the stability of the SMRS, the description of the stability is related to the changes of the pose of each module. Therefore, the coupling effect between the trajectory of each joint and the pose of each module should be clarified by the velocity-level kinematic model of SMRS. At the same time, the real-time pose of each joint in the velocity-level kinematic model needs to be calculated using the position-level kinematic model. In this section, the kinematic model of velocity-level and position-level of SMRS is established, respectively.

2.1. Velocity-Level Kinematic Model. Firstly, the meaning of the symbols in the kinematic modeling process of SMRS is defined and summarized in Table 1. The two joints of SMRS are connected by a connecting rod. At this time, the module can also be regarded as a connecting rod with a special shape. Usually, the attitude control module is selected to establish the base coordinate system of SMRS, and the base coordinate system is used to divide the satellite structure of SMRS into $a$ and $b$ sides. The structure diagram of SMRS is established as shown in Figure 2.

In Figure 2, $B_{0}$ represents the selected base; $B_{i}^{a}, i=1$ $\cdots n_{a}$, represents the connecting rods located in the $a$ side of $\sum_{b}, B_{i}^{b}, i=1 \cdots n_{b}$, represents the connecting rods located in the $b$ side of $\sum_{b}$; and $J_{i}^{a}$ and $J_{i}^{b}$ represent the $i$ th joint on both $a$ and $b$ sides, respectively. $n_{a}$ and $n_{b}$ are the numbers of joints on the $a$ and $b$ side, respectively. Each connecting rod has one center of mass coordinate system whose origin is on the connecting rod's center of mass and axes are parallel to $\sum_{I}$.

According to reference [17], the position of $C_{i}^{k},(k=a, b)$ in SMRS can be expressed by

$$
\mathbf{r}_{i}^{k}=\mathbf{r}_{0}+\mathbf{b}_{0}^{k}+\sum_{n=1}^{i}\left(\mathbf{a}_{n}^{k}+\mathbf{b}_{n}^{k}\right)-\mathbf{b}_{i}^{k}, \quad k=a, b .
$$




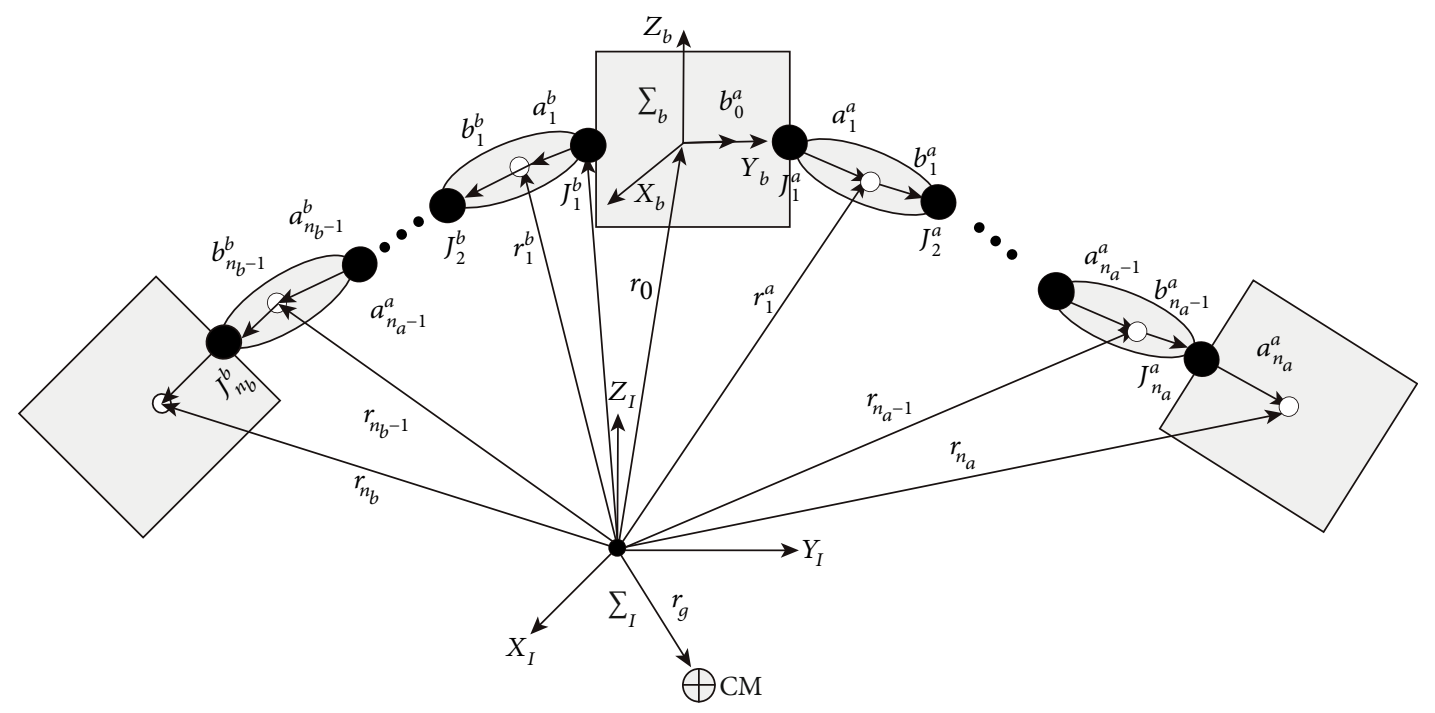

FIgURE 2: Schematic diagram of SMRS.

Deriving the time $t$ of Equation (1), the velocity of each connecting rod is obtained in the following equation:

$\mathbf{v}_{i}^{k}=\dot{\mathbf{r}}_{i}^{k}=\mathbf{v}_{b}+\boldsymbol{\omega}_{b}^{k} \times\left(\mathbf{r}_{i}^{k}-\mathbf{r}_{0}\right)+\sum_{n=1}^{\mathbf{i}}\left[\mathbf{k}_{n}^{k} \times\left(\mathbf{r}_{i}^{k}-\mathbf{p}_{n}^{k}\right)\right] \dot{\theta}_{n}^{k}, \quad k=a, b$.

In addition, the relationship between the angular velocity of each connecting rod and the rotational angular velocity of each joint is shown in the following equation:

$$
\boldsymbol{\omega}_{i}^{k}=\boldsymbol{\omega}_{b}+\sum_{n=1}^{i} \dot{\theta}_{n}^{k} \mathbf{k}_{n}^{k}, \quad k=a, b .
$$

Equations (2) and (3) are arranged into a matrix form, and the velocity vector of the $i$ th connecting rod on both the $a$ and $b$ sides is obtained:

$$
\left[\begin{array}{c}
\dot{\mathbf{x}}_{\mathrm{ii}}^{a} \\
\dot{\mathbf{x}}_{i}^{b}
\end{array}\right]=\left[\begin{array}{c}
\mathbf{J}_{b i}^{a} \\
\mathbf{J}_{b i}^{b}
\end{array}\right]\left[\begin{array}{c}
\mathbf{v}_{0} \\
\boldsymbol{\omega}_{0}
\end{array}\right]+\left[\begin{array}{cc}
\mathbf{J}_{m i}^{a} & 0 \\
0 & \mathbf{J}_{m i}^{b}
\end{array}\right]\left[\begin{array}{c}
\dot{\boldsymbol{\Theta}}^{a} \\
\dot{\Theta}^{b}
\end{array}\right],
$$

where $\dot{\mathbf{x}}_{i}^{a}=\left[\mathbf{v}_{i}^{a} \boldsymbol{\omega}_{i}^{a}\right]^{T}, \dot{\mathbf{x}}_{i}^{b}=\left[\begin{array}{ll}\mathbf{v}_{i}^{b} & \boldsymbol{\omega}_{i}^{b}\end{array}\right]^{T}$ are pose vectors of the $i$ th connecting rod on the $a$ and $b$ side.

$$
\boldsymbol{\Theta}^{k}=\left[\theta_{1}^{k}, \theta_{2}^{k}, \cdots, \theta_{n_{k}-1}^{k}, \theta_{n_{k}}^{k}\right], \quad k=a, b .
$$

$\mathbf{J}_{b i}=\left[\begin{array}{ll}\mathbf{J}_{b i}^{a} & \mathbf{J}_{b i}^{b}\end{array}\right]^{T}$ is the Jacobian matrix related to the motion of $\sum_{b}$;

$$
\mathbf{J}_{m i}=\left[\begin{array}{cc}
\mathbf{J}_{m i}^{a} & 0 \\
0 & \mathbf{J}_{m i}^{b}
\end{array}\right]
$$

is the Jacobian matrix related to the motion of the connecting rod.

$$
\begin{gathered}
\mathbf{J}_{b i}^{k}=\left(\begin{array}{cc}
\mathbf{E}_{3} & -\tilde{\mathbf{r}}^{\mathbf{k}}{ }_{0 i} \\
\mathbf{0} & \mathbf{E}_{3}
\end{array}\right)=\left[\begin{array}{c}
\mathbf{J}_{b v}^{k} \\
\mathbf{J}_{b w}^{k}
\end{array}\right] \in \mathbf{R}^{6 \times 6}, \quad k=a, b, \\
\mathbf{J}_{b i}^{k}=\left(\begin{array}{cc}
\mathbf{E}_{3} & -\tilde{\mathbf{r}}_{0 i}^{k} \\
\mathbf{0} & \mathbf{E}_{3}^{k}
\end{array}\right)=\left[\begin{array}{cc}
\mathbf{J}_{b v}^{k} \\
\mathbf{J}_{b w}^{k}
\end{array}\right] \in \mathbf{R}^{6 \times 6}, \quad k=a, b, \\
\mathbf{J}_{m i}^{k}=\left[\begin{array}{ccc}
\mathbf{k}_{1}^{k} \times\left(\mathbf{r}_{i}^{k}-\mathbf{p}_{1}^{k}\right), \mathbf{k}_{2}^{k} \times\left(\mathbf{r}_{i}^{k}-\mathbf{p}_{2}^{k}\right) & \cdots & \mathbf{k}_{i}^{k} \times\left(\mathbf{r}_{i}^{k}-\mathbf{p}_{i}^{k}\right), 0, \cdots, 0 \\
\mathbf{k}_{1}^{k}, \mathbf{k}_{2}^{k}, & \cdots & \mathbf{k}_{i}^{k}, 0, \cdots, 0
\end{array}\right], \quad k=a, b .
\end{gathered}
$$

When $\mathbf{x}=\left[\begin{array}{lll}x_{1}, & x_{2}, & x_{3}\end{array}\right]^{T}$, then

$$
\tilde{\mathbf{x}}=\left[\begin{array}{ccc}
0 & -x_{3} & x_{2} \\
x_{3} & 0 & -x_{1} \\
-x_{2} & x_{1} & 0
\end{array}\right] .
$$

In the free-floating state of SMRS, the pose of the space system is not controlled during the reconfiguration which can minimize the energy consumption. Simultaneously, assuming no other external forces and torques act on SMRS, in this state, the position of the CM will remain unchanged in inertia space. Therefore, we choose the CM as origin of $\Sigma_{I}$ to make $\mathbf{r}_{g}=0$ to simplify calculation. In addition, the whole SMRS satisfies the condition of conservation of momentum; that is, both the linear momentum and the angular momentum are conserved. Assuming initial momentum of SMRS is 0, its conservation equations of linear momentum and angular momentum are, respectively, shown in Equations (9) and (10).

$$
\begin{aligned}
\mathbf{P} & =\sum_{i=1}^{n_{a}+n_{b}} m_{i} \dot{\mathbf{r}}_{i}=m_{0} \dot{\mathbf{r}}_{0}+\sum_{i=1}^{n_{a}} m_{i}^{a} \dot{\mathbf{r}}_{i}^{a}+\sum_{i=1}^{n_{b}} m_{i}^{b} \dot{\mathbf{r}}_{i}^{b}=0, \\
\mathbf{L} & =\sum_{i=0}^{n_{a}+n_{b}}\left(\mathbf{I}_{i} \boldsymbol{\omega}_{i}+\mathbf{r}_{i} \times m_{i} \dot{\mathbf{r}}_{i}\right)=0 .
\end{aligned}
$$


Defining the angular momentum relative to $\Sigma_{b}$ center of mass as $\mathbf{L}_{0}$, the angular momentum of SMRS is $\mathbf{L}=\mathbf{L}_{0}$ $+\mathbf{r}_{0} \times \mathbf{P}$. According to the law of conservation of momentum, $\mathbf{L}=\mathbf{P}=\mathbf{O}$. Therefore $\mathbf{L}_{0}=\mathbf{O}$, the momentum conservation equation of SMRS is transformed into

$$
\begin{aligned}
{\left[\begin{array}{c}
\mathbf{P} \\
\mathbf{L}_{0}
\end{array}\right]=} & {\left[\begin{array}{cc}
\mathbf{M E} & \mathbf{M}^{T}{ }_{0 g} \\
\mathbf{M}_{0 g} & \mathbf{H}_{\omega}
\end{array}\right]\left[\begin{array}{c}
\mathbf{v}_{0} \\
\boldsymbol{\omega}_{0}
\end{array}\right] } \\
& +\left[\begin{array}{c}
\mathbf{J}_{T \omega}^{a} \\
\mathbf{H}_{\omega \varphi}^{a}
\end{array}\right] \dot{\boldsymbol{\Theta}}^{a}+\left[\begin{array}{c}
\mathbf{J}_{T \omega}^{b} \\
\mathbf{H}_{\omega \varphi}^{b}
\end{array}\right] \dot{\boldsymbol{\Theta}}^{b}=\mathbf{O} .
\end{aligned}
$$

In Equation (11),

$$
\begin{aligned}
\mathbf{H}_{\omega} & =\sum_{k=1}^{a, b} \sum_{i=1}^{n_{\mathrm{k}}}\left(\mathbf{I}_{i}^{k}+m_{i}^{k} \tilde{\mathbf{r}}_{i}^{\mathrm{k}}\left(\tilde{\mathbf{r}}_{0 i}^{k}\right)^{T}\right)+\mathbf{I}_{0} \in \mathbf{R}^{3 \times 3}, \quad k=a, b, \\
\mathbf{J}_{T \omega}^{k} & =\sum_{i=1}^{n}\left(m_{i}^{k} \mathbf{J}_{T i}^{k}\right), \quad k=a, b, \\
\mathbf{H}_{\omega \varphi}^{k} & =\sum_{i=1}^{n_{k}}\left(\mathbf{I}_{i}^{k} \mathbf{J}_{R i}^{k}+m_{i}^{k} \tilde{\mathbf{r}}_{i}^{k} \mathbf{J}_{T i}^{k}\right) \in \mathbf{R}^{3 \times n_{k}}, \quad k=a, b .
\end{aligned}
$$

For rotating joint,

$$
\begin{aligned}
\mathbf{J}_{T i}^{k} & =\left[\mathbf{k}_{1}^{k} \times\left(\mathbf{r}_{i}^{k}-\mathbf{p}_{1}^{k}\right), \cdots, \mathbf{k}_{i}^{k} \times\left(\mathbf{r}_{i}^{k}-\mathbf{p}_{i}^{k}\right), 0, \cdots, 0\right] \in \mathbf{R}^{3 \times n_{k}}, \quad k=a, b, \\
\mathbf{J}_{R i}^{k} & =\left[\mathbf{k}_{1}^{k}, \mathbf{k}_{2}^{k}, \cdots, \mathbf{k}_{i}^{k}, 0, \cdots 0\right] \in \mathbf{R}^{3 \times n_{k}}, \quad k=a, b .
\end{aligned}
$$

$\mathbf{r}_{0 i}=\mathbf{r}_{i}-\mathbf{r}_{0}, \mathbf{r}_{0 \mathrm{~g}}$ is the vector from the origin of $\Sigma_{b}$ to the CM; then, the momentum conservation equation can be further simplified to obtain the relationship between the linear velocity, angular velocity, and joint rotation angle of the base as shown in the following equation:

$$
\dot{\mathbf{x}}_{0}=\left[\begin{array}{c}
\mathbf{v}_{0} \\
\boldsymbol{\omega}_{0}
\end{array}\right]=-\left[\begin{array}{cc}
\mathbf{M E} & \mathbf{M} \tilde{\mathbf{r}}^{T} \\
\mathbf{M}_{0 g} & \mathbf{H}_{\omega}
\end{array}\right]^{-1}\left(\left[\begin{array}{c}
\mathbf{J}_{T \omega}^{a} \\
\mathbf{H}_{\omega \varphi}^{a}
\end{array}\right] \dot{\boldsymbol{\Theta}}^{a}+\left[\begin{array}{c}
\mathbf{J}_{T \omega}^{b} \\
\mathbf{H}_{\omega \varphi}^{b}
\end{array}\right] \dot{\boldsymbol{\Theta}}^{b}\right) .
$$

Arranging Equation (14), we obtain

$$
\dot{\mathbf{x}}_{0}=\mathbf{J}_{b m}^{a} \dot{\boldsymbol{\Theta}}^{a}+\mathbf{J}_{b m}^{b} \dot{\boldsymbol{\Theta}}^{b}=\left[\begin{array}{ll}
\mathbf{J}_{b m}^{a} & \mathbf{J}_{b m}^{b}
\end{array}\right]\left[\begin{array}{c}
\dot{\boldsymbol{\Theta}}^{a} \\
\dot{\boldsymbol{\Theta}}^{b}
\end{array}\right]
$$

$\mathbf{J}_{b m}^{k}$ is the Jacobian matrix of the base and joint trajectories. Equation (15) shows the coupling effect between the pose of the base and the joint motion.
Take Equation (15) into Equation (4), we can obtain

$$
\left[\begin{array}{c}
\dot{\mathbf{x}}_{i}^{a} \\
\dot{\mathbf{x}}_{i}^{b}
\end{array}\right]=\left(\left[\begin{array}{c}
\mathbf{J}_{b i}^{a} \\
\mathbf{J}_{b i}^{b}
\end{array}\right]\left[\begin{array}{ll}
\mathbf{J}_{b m}^{a} & \mathbf{J}_{b m}^{b}
\end{array}\right]+\left[\begin{array}{cc}
\mathbf{J}_{m i}^{a} & 0 \\
0 & \mathbf{J}_{m i}^{b}
\end{array}\right]\right)\left[\begin{array}{c}
\dot{\boldsymbol{\Theta}}^{\mathrm{a}} \\
\dot{\boldsymbol{\Theta}}^{b}
\end{array}\right]=\mathbf{J}_{g i}\left[\begin{array}{c}
\dot{\boldsymbol{\Theta}}^{a} \\
\dot{\Theta}^{b}
\end{array}\right] .
$$

$\mathbf{J}_{g i} \in \mathbf{R}^{12 \times\left(n_{a}+n_{b}\right)}$ is a generalized Jacobian matrix (GJM) about the pose of each connecting rod, joint trajectories, and mass characteristics of SMRS. It represents the dynamic coupling effect between the motion of each joint and the pose of each connecting rod and proves that the motion of any joint will affect the pose of every connecting rod.

2.2. Position-Level Kinematic Model. The velocity-level kinematic model of SMRS is derived above, and the Jacobian matrix related to the connecting rod's velocity and joint trajectories is obtained. The calculation of the Jacobian matrix needs the real-time pose of each connecting rod which is described by the position-level kinematic model. Therefore, it is necessary to deduce the position-level kinematic model of SMRS.

Among the various kinematic modeling methods of multiconnecting rod systems, the Denavit-Hartenberg method (D-H) [18] is not only suitable to establish the coordinates of the multiconnecting rod system but also simple and fast to iterative calculate the connecting rod poses using multiconnecting rod homogeneous transformation. Moreover, the $\mathrm{D}-\mathrm{H}$ method can be used for Jacobian matrix analysis and dynamic calculations. Therefore, this paper adopts the $\mathrm{D}-\mathrm{H}$ method to establish the coordinate system of SMRS and calculate the pose of each connecting rod. According to the principles of establishing the coordinate system in the $\mathrm{D}-\mathrm{H}$ method, each joint is fixed with a joint coordinate system; then, the coordinate calculation can be performed between the coordinate systems through the homogeneous matrix transformation. Through successive transformations of each joint's coordinate system, the pose of joints and connecting rods can also be derived.

The D-H method selects four parameters between adjacent coordinate systems to describe the relationship of the coordinate system; they are link length $A$; link twist $\alpha$, link offset $d$, and joint angle $\theta$. The four parameters of $\mathrm{D}-\mathrm{H}$ are referenced to the current coordinate system to perform a determined four-step transformation to obtain a transformation matrix from the $(i-1)$ th coordinate system to the $i$ th coordinate system:

$$
{ }_{i}^{i-1} R=\left[\begin{array}{cccc}
\cos \theta_{i-1} & -\sin \theta_{i} & 0 & A_{i-1} \\
\sin \theta_{i} c \alpha_{i-1} & \cos \theta_{i} c \alpha_{i-1} & -\sin \alpha_{i-1} & -\sin \alpha_{i-1} d_{i} \\
\sin \theta_{i} \sin \alpha_{i-1} & \cos \theta_{i} s \alpha_{i-1} & \cos \alpha_{i-1} & \cos \alpha_{i-1} d_{i} \\
0 & 0 & 0 & 1
\end{array}\right] .
$$

If there is a point $P$ in space whose coordinate in the $i$ th coordinate system is $p_{i}$ and its coordinate in the $(i-1)$ th coordinate system is $p_{i-1}$, then the relationship between $p_{i}$ and $p_{i-1}$ can be expressed as 


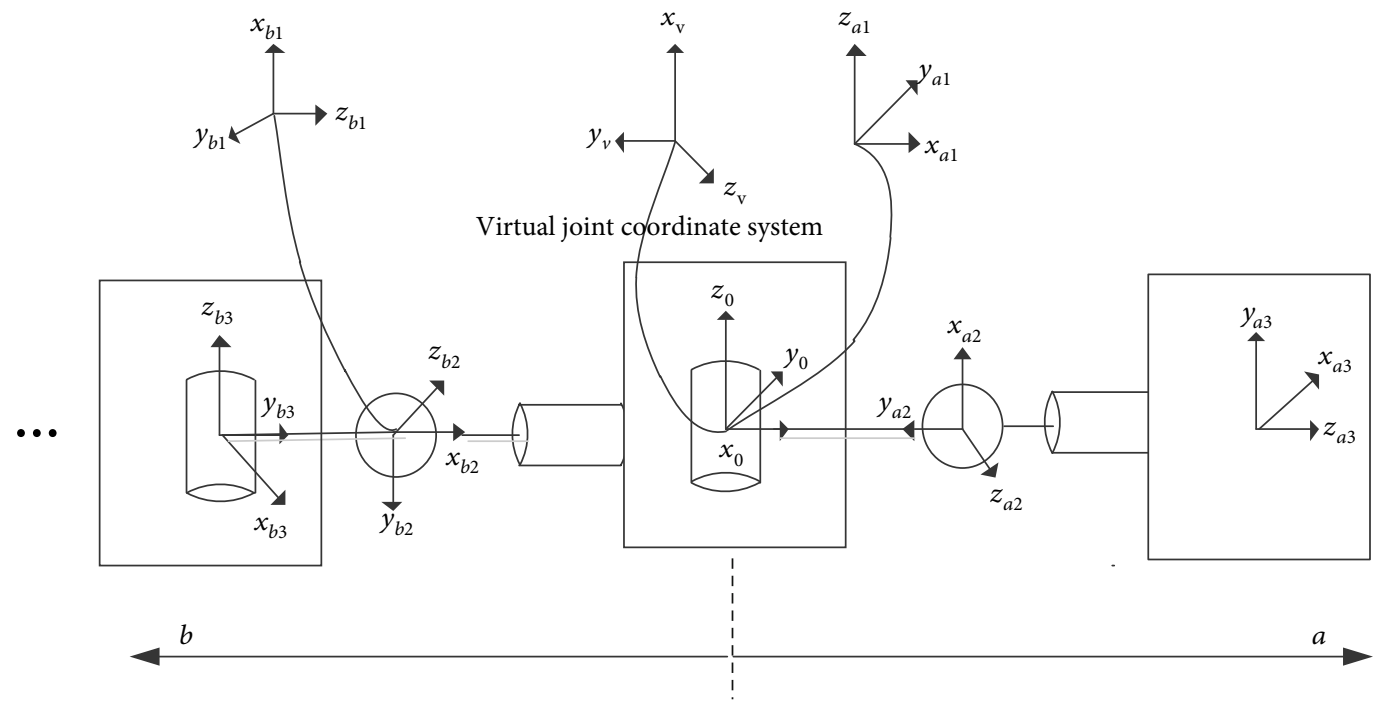

Figure 3: Coordinate system of SMRS.

$$
p_{i-1}={ }_{i}^{i-1} R p_{i}
$$

After $i$ times of transformations, the pose of the $i$ th connecting rod can be reached, as shown in Equation (19), and the total transformation matrix between the base and $i$ th connecting rod is obtained by multiplying the transformation matrices between adjacent coordinate systems.

$$
{ }_{i}^{0} R={ }_{1}^{0} R_{2}^{1} R_{3}^{2} \cdots{ }_{i}^{i-1} R \text {. }
$$

The first step of establishing the SMRS's kinematic model is to define the coordinate system. The $\Sigma_{b}$ of SMRS is established in the center of mass of attitude control module, and the connecting rod coordinate systems in two sides ( $a$ side, $b$ side) are established, respectively, on either side of $\Sigma_{b}$. In some space systems such as space dual-arm robots, the joint axis orientations on both sides of $\Sigma_{b}$ are always consistent during design, so the $\mathrm{D}-\mathrm{H}$ parameters on both sides are equal. However, the three joints between the modules of the SMRS are series designed, and they are orthogonal to each other. Meanwhile, it is not possible to make special designs for the joints on both sides of the base coordinate system to keep their direction consistent because the position of $\Sigma_{b}$ is not fixed. As shown in Figure 3, when the first joint coordinate system on the $a$ side is completely coincident with the base coordinate system, its $x$-axis is perpendicular to the $z$ -axis of the second joint on the $a$ side; however, the first joint axis on the $b$ side is orthogonal to the joint axes of 1 and 2 on the $a$ side, so that the $x$-axis of $\Sigma_{b}$ is parallel to the first joint axis on the $b$ side which violates the principle of establishing the coordinate system in the D-H method.

To solve the problem, a virtual joint coordinate system is proposed. Virtual joint coordinate system Jv is a coordinate system established between the base coordinate system and the first joint coordinate system on the $b$ side to solve the problem that the $x$-axis of $\Sigma_{b}$ is parallel to the $z$-axis of the first joint on the $b$ side. The D-H coordinate systems of SMRS and virtual joint coordinate system are shown in Figure 3.

The standard for establishing the virtual joint coordinate system is as follows: the $z$-axis of the virtual joint coordinate system is perpendicular to the $x$-axis of $\Sigma_{b}$ and the $z$-axis of the first joint coordinate system on the $b$ side, and the $x$-axis is perpendicular to the $z$-axis of the first joint coordinate system on the $b$ side. At the same time, the origin of the virtual joint coordinate system coincides with the origin of $\Sigma_{b}$. Because of the introduction of the virtual joint coordinate system, the degree of freedom of SMRS is increased by 1 . When mass and structural size are set to 0 and remain always static, the virtual joint does not affect the quality parameters and dimensions of the system. Then, the calculation of the pose matrix of the connecting rod on the $b$ side only needs to multiply the transformation matrix from the virtual joint coordinate system to the base coordinate system between the first joint coordinate system and $\Sigma_{b}$.

\section{Trajectory Optimization of SMRS}

3.1. Cost Function and Constraints. According to the derivation in Section 2, it can be known that the pose of base is affected by the trajectories of joints. Because of the functional characteristics, SMRS has the specific configuration in a specific space missions, which requires every joint to move to a certain angle within a certain mission time. Due to the conservation of momentum in space, the total pose changes of connecting rods relative to $\Sigma_{I}$ are constant values from the current configuration to the target configuration. It means the joint trajectories would not affect the mean values of the connecting rod's pose velocity curves. What would be affected by joint trajectories are the shapes of pose velocity curves, which are closely related to the movement stability of SMRS. The improvement of movement stability of SMRS is supposed to provide a more stable state for important instruments carried in satellite modules and avoid sudden torque changes between joints. 
Therefore, improving the movement stability of SMRS in the reconfiguration process is taken as the optimization objective of joint trajectory optimization. When the pose velocity curves of connecting rods are continuous and smooth, their movement stability can be characterized by the pose velocity extremums. The movement stability of SMRS depends on pose velocities of all connecting rods. Nevertheless, to reduce the complexity of the cost function, we choose the sum of the absolute extremums of all linear and angular velocities of one or several selected modules to characterize the movement stability of SMRS. The selected module can be different by different evaluation standards. At this time, the cost function of this optimization problem can be defined in the following equation:

$$
\Gamma(\boldsymbol{\Theta})=\sum_{s=1}^{g}\left(\sum_{i=1}^{6} \max \left|\dot{\mathbf{x}}_{s}(i, 1)\right|\right)
$$

where $g$ is the number of selected modules and $\dot{\mathbf{x}}_{s} \in R^{6 \times 1}, s=1 \cdots g$, are velocity vectors of them.

The joint trajectories directly affect the stability of the actuator output torques [19]. In order to ensure the smoothness of the joint trajectories in SMRS, the initial value, end value, velocity, and acceleration of each joint trajectory need to be constrained to satisfy Equations (21)-(32).

$$
\begin{gathered}
\boldsymbol{\Theta}^{a}\left(t_{\mathrm{s}}\right)=\boldsymbol{\Theta}_{0}^{a}, \\
\boldsymbol{\Theta}^{b}\left(t_{s}\right)=\boldsymbol{\Theta}_{0}^{b}, \\
\boldsymbol{\Theta}^{a}\left(t_{f}\right)=\boldsymbol{\Theta}_{f}^{a}, \\
\boldsymbol{\Theta}^{b}\left(t_{f}\right)=\boldsymbol{\Theta}_{f}^{b}, \\
\dot{\boldsymbol{\Theta}}^{a}\left(t_{s}\right)=\ddot{\boldsymbol{\Theta}}^{a}\left(t_{s}\right)=0, \\
\dot{\boldsymbol{\Theta}}^{b}\left(t_{s}\right)=\ddot{\boldsymbol{\Theta}}^{b}\left(t_{s}\right)=0, \\
\dot{\boldsymbol{\Theta}}^{a}\left(t_{f}\right)=\ddot{\boldsymbol{\Theta}}^{a}\left(t_{f}\right)=0, \\
\dot{\boldsymbol{\Theta}}^{b}\left(t_{f}\right)=\ddot{\boldsymbol{\Theta}}^{b}\left(t_{f}\right)=0, \\
\boldsymbol{\Theta}_{\min }^{k} \leq \boldsymbol{\Theta}^{k} \leq \boldsymbol{\Theta}_{\max }^{k}, \\
\dot{\boldsymbol{\Theta}}_{\min }^{k} \leq \dot{\boldsymbol{\Theta}}^{k} \leq \dot{\boldsymbol{\Theta}}_{\max }^{k}, \\
\ddot{\boldsymbol{\Theta}}_{\min }^{k} \leq \ddot{\boldsymbol{\Theta}}^{k} \leq \ddot{\boldsymbol{\Theta}}_{\max }^{k}, \\
k=a, b,
\end{gathered}
$$

where $t_{s}$ and $t_{f}$ are the initial time and end time of the mission; $\Theta^{a}$ and $\Theta^{b}$ are the joint angle vectors of both sides of $a$ and $b$, respectively; $\boldsymbol{\Theta}_{0}^{a}$ and $\boldsymbol{\Theta}_{f}^{a}$ are the joint angle vector of the $a$ side at initial and end time, respectively. $\boldsymbol{\Theta}_{0}^{b}$ and $\boldsymbol{\Theta}_{f}^{b}$ are the joint angle vector of $b$ side at initial and end time, respectively. $\boldsymbol{\Theta}_{\max }^{k}, \dot{\Theta}_{\max }^{k}, \ddot{\Theta}_{\text {max }}^{k}, k=a, b$, are the vector of maximum joint angle allowed, maximum joint angular velocity, and maximum joint angular acceleration. $\Theta_{\text {min }}^{k}, \dot{\Theta}_{\text {min }}^{k}, \ddot{\Theta}_{\text {min }}^{k}, k=a, b$, are the vector of minimum joint angle, minimum joint angular velocity, and minimum joint angular acceleration allowed.

3.2. Parameterization of Joint Trajectory. In order to ensure the above constraints in Equations (21)-(32) and define the control parameters, the joint trajectory is usually parameterized by some curves which include polynomials [20-22], trigonometric functions, Bezier curves [23], and spline difference curves $[24,25]$. Among them, the polynomial is an ideal parameterized curve because of its simple form. At the same time, its order can be changed according to the joint differential constraints of different orders, and the number of control parameters can be arbitrarily selected. In this paper, a polynomial whose general form is expressed by Equation (33) is used to parameterize the joint trajectories.

$$
\theta_{i}^{k}(t)=a_{\mathrm{in}}^{k} t^{n}+a_{i(n-1)}^{k} t^{n-1}+\cdots a_{i 1}^{k} t+a_{i 0}^{k}, \quad k=a, b .
$$

While the constraints for continuity and smoothness of velocity, acceleration, and smoothness are satisfied in Equations (21)-(28), the minimum order of the polynomial is 5 . Considering fewer control parameters can accelerate the convergence of the optimization process, 6-order and 7-order polynomials which have only one and two control parameters, respectively, are chosen to parameterize the joint trajectories.

(1) Use Equation (33) to parameterize the 6-order polynomial for each joint trajectory on both sides of $a$ and $b$. At this time, $n=6$, set $t=0$ as the initial time of joint motion, and bring the constraints in Equations (21)-(28) into Equation (33) to obtain

$$
\begin{aligned}
& a_{i 0}^{k}=\theta_{i 0}^{k} ; a_{i 1}^{k}=\dot{\theta}_{i 0}^{k} ; a_{i 2}^{k}=\frac{1}{2} \ddot{\theta}_{i 0}^{k}, k=a, b, \\
& a_{i 3}^{k}=-\left(20 \theta_{0}^{k}-20 \theta_{f}^{k}+12 \dot{\theta}_{0}^{k} t_{f}+8 \dot{\theta}_{f}^{k} t_{f}+2 a_{i 6}^{k} t_{f}^{6}+12 \ddot{\theta}_{0}^{k} t_{f}^{2} \ddot{\theta}_{f}^{k} t_{f}^{2}\right) / 2 t_{f}^{3}, \quad k=a, b, \\
& a_{i 4}^{k}=\frac{\left(15 \theta_{0}^{k}-15 \theta_{f}^{k}+8 \dot{\theta}_{0}^{k} t_{f}+7 \dot{\theta}_{f}^{k} t_{f}+3 a_{i 6}^{k} t_{f}^{6}+6 \ddot{\theta}_{0}^{k} t_{f}^{2}-\ddot{\theta}_{f}^{k} t_{f}^{2}\right)}{t_{f}^{4}}, \quad k=a, b,
\end{aligned}
$$

$$
a_{i 5}^{k}=\frac{-\left(12 \theta_{0}^{k}-12 \theta_{f}^{k}+6 \dot{\theta}_{0}^{k} t_{f}+6 \dot{\theta}_{f}^{k} t_{f}+6 a_{i 6}^{k} t_{f}^{6}+4 \ddot{\theta}_{0}^{k} t_{f}^{2}-\ddot{\theta}_{f}^{k} t_{f}^{2}\right)}{2 t_{f}^{5}}, \quad k=a, b
$$

From Equations (34)-(37), we know that the parameterized $i$ th joint trajectory can be written as an equation with polynomial coefficients $a_{i 6}^{k}, k=a, b$, as parameters, and its 
specific form is shown in the following equation:

$$
\begin{aligned}
\theta_{i}^{k}(t)= & a_{i 6}^{k} t^{6}+\left(-30 a_{i 6}^{k}-\frac{3}{5000}\right) t^{5}+\left(300 a_{i 6}^{k}+\frac{3}{200}\right) t^{4} \\
& +\left(-1000 a_{i 6}^{k}-\frac{1}{10}\right) t^{3}+\theta_{i 0}^{k}+\dot{\theta}_{i 0}^{k}+\frac{1}{2} \ddot{\theta}_{i 0}^{k}, \quad k=a, b .
\end{aligned}
$$

According to Equation (38), when the constraints in Equations (21)-(32) remain unchanged, the trajectory curve can be adjusted by changing the polynomial coefficient $a_{i 6}^{k}$, $k=a, b$, of the $i$ th joint trajectory. It means that $a_{i 6}^{k}, k=a, b$, can be seen as the trajectory control parameter to optimize cost function. Since each joint trajectory has one control parameter after parameterization, the optimization vector on the $a$ or $b$ side of the system is determined as $\left[a_{16}^{k}, a_{26}^{k}, \cdots\right.$ ,$\left.a_{n_{k} 6}^{k}\right], k=a, b$.

(2) Use Equation (33) to parameterize the 7-order polynomial for each joint trajectory on both sides of $a$ and $b$. At this time, $n=7$, set $t=0$ as the initial time of joint motion, and bring the constraints in Equations (21)-(28) into Equation (33) to obtain

$$
\begin{gathered}
a_{i 0}^{k}=\theta_{i 0}^{k} ; a_{i 1}^{k}=\dot{\theta}_{i 0}^{k} ; a_{i 2}^{k}=\frac{1}{2} \ddot{\theta}_{i 0}^{k}, \quad k=a, b, \\
a_{i 3}^{k}=-\left(20 \theta_{0}^{k}-20 \theta_{f}^{k}+12 \dot{\theta}_{0}^{k} t_{f}+8 \dot{\theta}_{f}^{k} t_{f}+2 a_{i 6}^{k} t_{f}^{6}+2 a_{i 7}^{k} t_{f}^{7}+12 \ddot{\theta}_{0}^{k} t_{f}^{2}-\ddot{\theta}_{f}^{k} t_{f}^{2}\right) / 2 t_{f}^{3}, \quad k=a, b, \\
a_{i 4}^{k}=\left(15 \theta_{0}^{k}-15 \theta_{f}^{k}+8 \dot{\theta}_{0}^{k} t_{f}+7 \dot{\theta}_{f}^{k} t_{f}+3 a_{i 6}^{k} t_{f}^{6}+8 a_{i 6}^{k} t_{f}^{7}+6 \ddot{\theta}_{0}^{k} t_{f}^{2}-\ddot{\theta}_{f}^{k} t_{f}^{2}\right) / t_{f}^{4}, \quad k=a, b, \\
a_{i 5}^{k}=-\left(12 \theta_{0}^{k}-12 \theta_{f}^{k}+6 \dot{\theta}_{0}^{k} t_{f}+6 \dot{\theta}_{f}^{k} t_{f}+6 a_{i 6}^{k} t_{f}^{6}+12 a_{i 7}^{k} t_{f}^{7}+4 \ddot{\theta}_{0}^{k} t_{f}^{2}-\ddot{\theta}_{f}^{k} t_{f}^{2}\right) / 2 t_{f}^{5}, \quad k=a, b
\end{gathered}
$$

From Equations (39)-(42), we know that the parameterized $i$ th joint trajectory can be written as an equation with polynomial coefficients $a_{i 6}^{k}, a_{i 7}^{k}, k=a, b$, as parameters, and its specific form is shown in the following equation (43):

$$
\begin{aligned}
\theta_{i}^{k}(t)= & a_{i 7}^{k} t^{7}+a_{i 6}^{k} t^{6}+\left(-21600 a_{i 7}^{k}-180 a_{i 6}^{k}+\frac{1}{43200000}\right) t^{5} \\
& +\left(172800 a_{i 7}^{k}+10800 a_{i 6}^{k}-\frac{1}{288000}\right) * t^{4} \\
& +\left(-3888000 a_{i 7}^{k}-216000 a_{i 6}^{k}+\frac{1}{7200}\right) t^{3} \\
& +\theta_{i 0}^{k}+\dot{\theta}_{i 0}^{k}+\frac{1}{2} \ddot{\theta}_{i 0}^{k}, \quad k=a, b .
\end{aligned}
$$

According to Equation (43), when the constraints in Equations (21)-(32) remain unchanged, the trajectory curve can be adjusted by changing the polynomial coefficient $a_{i 6}^{k}$, $a_{i 7}^{k}, k=a, b$, of the $i$ th joint trajectory. It means that $a_{i 6}^{k}, a_{i 7}^{k}$, $k=a, b$, can be seen as the trajectory control parameters to optimize the cost function. Since each joint trajectory of SMRS has two control parameters after parameterization, the optimization vector on the $a$ or $b$ side of SMRS is determined as $\left[a_{16}^{k}, a_{26}^{k}, \cdots, a_{n_{k} 6}^{k}, a_{17}^{k}, a_{27}^{k}, \cdots, a_{n_{k}}^{k}\right], k=a, b$.

Introducing the parameterized joint trajectory into Equation (15), the relationship between control parameters and optimization objectives can be obtained based on the form of cost function in Equation (20). At this time, the optimiza- tion problem of the cost function is transformed into the optimization of control parameters.

\section{PSO Algorithm}

When the cost function and the optimization vector composed of the control parameters are determined, certain methods are needed to optimize the joint trajectory to achieve the optimization objective. Among all kinds of optimization methods, heuristic algorithm is widely used in joint trajectory planning, including simulated annealing (SA), genetic algorithm (GA), particle swarm optimization (PSO) [24], and ant colony optimization (ACO) [14] algorithm.

In this paper, the PSO algorithm is used to plan the joint trajectories of SMRS. The PSO algorithm is an intelligent optimization algorithm based on swarm intelligence [24], which is widely used to solve all kinds of engineering problems. Many researchers improve it or combine it with other algorithms to achieve better optimization results for different problems [26, 27]. Compared with other optimization algorithms, the PSO algorithm has the following superiorities: (1) few built-in parameters need to be adjusted; (2) it can find the optimal or approximate optimal solution of nonlinear and discontinuous problems in a higher dimension; (3) it is easy to realize by computer; (4) the unidirectional information flow makes the whole search and update process follow the current optimal solution, which makes the convergence speed faster [28].

The PSO algorithm first initializes a group of random particles of each optimization parameter and updates the fitness to find the optimal solution of the optimization 
parameter through each iteration. For the trajectory planning problem of SMRS, the fitness is the value of the cost function. Initially, the position of the $k$ th particle in each group of particles is set as $x_{k}$, and its initial velocity is $v_{k}$ [29]. The velocities and positions of all particles are updated iteratively according to Equation (44) and (45) to seek better fitness:

$$
\begin{aligned}
v_{k} & =w v_{k}+c_{1} a_{1}\left(p_{k}-x_{k}\right)+c_{2} a_{2}\left(p_{g}-x_{k}\right), \\
x_{k+1} & =x_{k}+v_{k} .
\end{aligned}
$$

In Equation (44), $w$ is inertia weight which controls the search field. $p_{k}$ is the local optimal position of the $k$ th particle. $p_{\mathrm{g}}$ is the global optimal position. $c_{1}$ and $c_{2}$ are learning factors usually in $[0.5,2] ; a_{1}$ and $a_{2}$ are random numbers. In the process of optimization, the particle position is evaluated by fitness, and the local optimal position $p_{k}$ of every particle so far and the global optimal position $p_{\mathrm{g}}$ of particle swarm so far are searched in search space. The motion of each particle is guided by $p_{k}$ and $p_{\mathrm{g}}$ which are updated in every generation. When we get the best fitness so far, that is to say, the particle position at this time is the better position found so far. This process is repeated generation by generation until the termination conditions are met. In this process, the $p_{k}$ and the $p_{\mathrm{g}}$ of all particles are two important values.

An important step in the PSO algorithm is to impose constraints on the optimization parameters. In this paper, the specific strategies to impose constraints on $x_{k}$ in the process of repairing infeasible solutions are as follows:

$$
x_{k}= \begin{cases}x_{\min }, & \text { if } x_{k} \leq x_{\min } \\ x_{\max }, & \text { if } x_{k} \geq x_{\max } \\ x_{k}, & \text { if otherwise }\end{cases}
$$

At the same time, the particle's velocity $v_{k}$ also needs to be constrained. If $v_{k}$ is too large, it is easy to cross the optimal solution. If the $v_{k}$ is too small, the convergence speed of the fitness is slow. The constraining strategy of $v_{k}$ is the same as that of $x_{k}$, as shown in the following equation:

$$
v_{k}= \begin{cases}v_{\min }, & \text { if } v_{k} \leq v_{\min }, \\ v_{\max }, & \text { if } v_{k} \geq v_{\max }, \\ v_{k}, & \text { if otherwise. }\end{cases}
$$

In Equations (46) and (47), $x_{\max }, x_{\min }, v_{\max }$, and $v_{\min }$ are the upper and lower limits set for $x_{k}$ and $v_{k}$. In the iteration of the PSO algorithm, the iteration termination condition of particles is often set as the maximum number of iterations or the optimal position so far has met the predetermined minimum fitness threshold. This article chooses maximum number of iterations iter $_{\max }$ as the termination condition.

The constraints of joint angle, velocity, and acceleration in Equation (29) is realized by penalty function in Equations (48)-(50) in the PSO algorithm.

$$
\begin{aligned}
& \text { fitness }=\left\{\begin{array}{l}
\text { fitness, } \\
\text { if } \boldsymbol{\Theta}_{\min }^{k} \leq \boldsymbol{\Theta}^{k} \leq \boldsymbol{\Theta}_{\max }^{k},
\end{array}\right. \\
& \text { fitness }= \begin{cases}\text { fitness, } & \text { if } \dot{\boldsymbol{\Theta}}_{\min }^{k} \leq \dot{\boldsymbol{\Theta}}^{k} \leq \dot{\boldsymbol{\Theta}}_{\max }^{k}, \\
\Delta, & \text { if otherwise, }\end{cases} \\
& \text { fitness }=\left\{\begin{array}{l}
\text { fitness, } \\
\Delta, \quad \text { if } \ddot{\boldsymbol{\Theta}}_{\min }^{k} \leq \ddot{\boldsymbol{\Theta}}^{k} \leq \ddot{\boldsymbol{\Theta}}_{\max }^{k},
\end{array}\right.
\end{aligned}
$$

In Equations (48)-(50), $\Delta$ can be any value far beyond the possible fitness. The current global optimal optimization parameters are brought into the trajectory curve to calculate the maximum value of joint angle, velocity, and acceleration. If they exceed the set intervals, the fitness will return to $\Delta$ and restart calculation. The flow of using the PSO algorithm to plan joint trajectories is shown in Figure 4.

\section{Trajectory Planning Simulation}

In this paper, SMRS composed of 9 connecting rods and 24 joints is selected as the research object. And the middle module is selected as the base module, and $\Sigma_{b}$ is fixed to it. Considering the base module usual carries the important subsystems such as a housekeeping computer, the cost function in Equation (20) is defined as the sum of absolute extremums of all linear and angular velocities of the base module. The parameters of the D-H coordinate system established by the initial state of linear SMRS are shown in Table 2. The connecting rod parameters of each joint of SMRS are shown in Table 3. The inertia weight $w=0.5$, learning factors $c_{1}=1.5$ and $c_{2}=1.5, \Delta=10000, \theta_{\text {max }}^{k}=90^{\circ}, \theta_{\text {min }}^{k}=-90^{\circ}, \dot{\theta}_{\max }^{k}=10^{\circ} / \mathrm{s}$, $\dot{\theta}_{\text {min }}^{k}=-10^{\circ} / s, \ddot{\theta}_{\text {max }}^{k}=2^{\circ} / s^{2}$, and $\ddot{\theta}_{\text {min }}^{k}=-2^{\circ} / s^{2}, k=a, b$, are set in the PSO algorithm. The iteration termination condition is chosen as the maximum number of iterations and is set to 100 .

5.1. Case 1. In order to reduce the occupied fairing space and facilitate the multistar package and launch, SMRS is usually the folded state shown in Figure 5(a) at launch and then unfold after getting into orbit. The unfolding process is shown in Figure 5(b), and the unfolded state is shown in Figure 5(c). In case 1, the trajectory planning of SMRS unfolding process is simulated while the initial time is set as 0 and the mission time as $60 \mathrm{~s}$.

The unfolded state of SMRS is a linear state which is also the state in which the coordinate system is established by the $\mathrm{D}-\mathrm{H}$ method. The joint angle vectors relative to the linear state are $\Theta_{0}^{a}=\left[0,0,0,90^{\circ}, 0,0,90^{\circ}, 0,0,0,0,0\right]$ of the $a$ side and $\Theta_{0}^{b}=\left[0,0,90^{\circ}, 0,0,90^{\circ}, 0,0,0,0,0,0\right]$ of the $b$ side. When SMRS is in the folded state, the unfolded state is the linear state, at this time, $\Theta_{f}^{k}=[0,0,0,0,0,0,0,0,0,0,0,0], k=a, b$. In order to ensure the stability of joint motion, the angular velocity and acceleration at the initial and end time of each joint angle are set to 0 . The range of control parameters is set as $\left[-1.0 \times 10^{-4}, 1.0 \times 10^{-4}\right]$ to ensure the monotonicity of trajectory curves. 


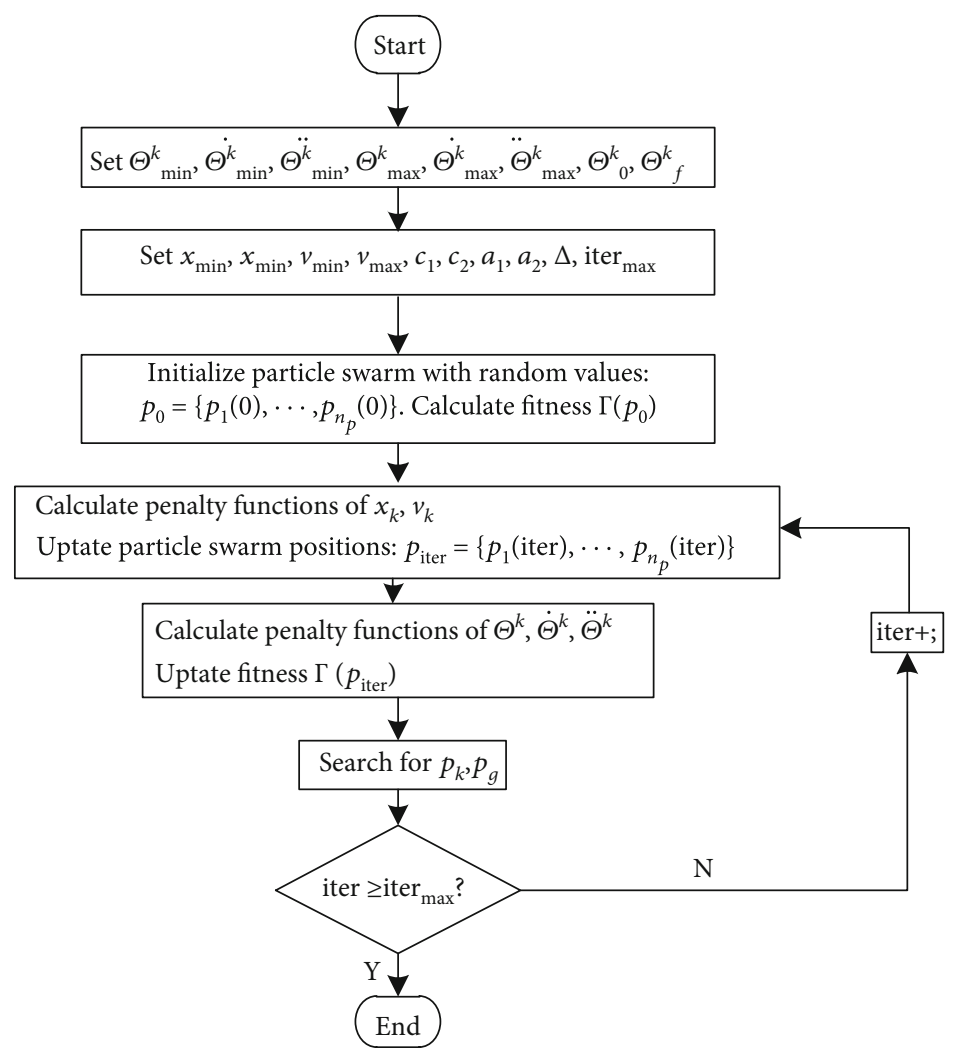

FIgURE 4: The flow chart of the PSO algorithm for trajectory planning.

TABLE 2: D-H parameters of SMRS.

\begin{tabular}{|c|c|c|c|c|c|c|c|c|c|}
\hline \multicolumn{5}{|c|}{ D-H parameters of the $a$ side } & \multicolumn{5}{|c|}{$\mathrm{D}$-H parameters of the $b$ side } \\
\hline Link $i$ & $\theta_{i}^{a}(\mathrm{deg})$ & $d_{i}^{a}(\mathrm{~m})$ & $\alpha_{i-1}^{a}(\mathrm{deg})$ & $A_{i-1}^{a}(\mathrm{~m})$ & Link $i$ & $\theta_{i}^{b}(\mathrm{deg})$ & $d_{i}^{b}(\mathrm{~m})$ & $\alpha_{i-1}^{b}(\mathrm{deg})$ & $A_{i-1}^{b}(\mathrm{~m})$ \\
\hline 1 & 0 & 0 & 0 & 0 & $\mathrm{v}^{1}-0$ & 90 & 0 & 90 & 0 \\
\hline 2 & 90 & 0 & 90 & 0.198 & $1-\mathrm{v}$ & 0 & -0.243 & 90 & 0 \\
\hline 3 & -90 & 0.243 & 90 & 0 & 2 & 90 & 0 & 90 & 0 \\
\hline 4 & -90 & 0 & -90 & 0 & 3 & -90 & 0 & 90 & -0.198 \\
\hline 5 & 90 & 0 & 90 & 0.198 & 4 & -90 & -0.243 & -90 & 0 \\
\hline 6 & -90 & 0.243 & 90 & 0 & 5 & 90 & 0 & 90 & 0 \\
\hline 7 & -90 & 0 & -90 & 0 & 6 & -90 & 0 & 90 & -0.198 \\
\hline 8 & 90 & 0 & 90 & 0.198 & 7 & -90 & -0.243 & -90 & 0 \\
\hline 9 & -90 & 0.243 & 90 & 0 & 8 & 90 & 0 & 90 & 0 \\
\hline 10 & -90 & 0 & -90 & 0 & 9 & -90 & 0 & 90 & -0.198 \\
\hline 11 & 90 & 0 & 90 & 0.198 & 10 & -90 & -0.243 & -90 & 0 \\
\hline 12 & -90 & 0.243 & 90 & 0 & 11 & 90 & 0 & 90 & 0 \\
\hline- & - & - & - & - & 12 & -90 & 0 & 90 & -1.32 \\
\hline
\end{tabular}

${ }_{\mathrm{V}}^{1}$ represents virtual joints.

While planning joint trajectories in the form of 6-order polynomials, the optimization parameter vector composed of 8 optimization parameters is $\left[a_{46}^{a}, a_{76}^{a}, a_{36}^{b}, a_{66}^{b}\right]$. While planning joint trajectories in the form of 7-order polynomial, the optimization parameter vector composed of 8 optimization parameters is $\left[a_{46}^{a}, a_{76}^{a}, a_{36}^{b}, a_{66}^{b}, a_{47}^{a}, a_{77}^{a}, a_{37}^{b}, a_{37}^{b}\right]$.

The PSO algorithm is used to optimize the trajectory parameters in the form of 6-order polynomial and 7-order polynomial, respectively, and their convergence process of fitness is shown in Figures 6(a) and 6(b).

The optimization results in Figure 6(a) show that the fitness value converges to 0.0484 based on the optimization vector which converges to $[0,0,0,0]$, and at this time, the highest order coefficient of the 6-order polynomial is 0 ; then, the polynomial is converted to the 5order polynomial. 
TABLE 3: Parameters of connecting rods of SMRS.

\begin{tabular}{lcccccccc}
\hline & $B_{0}$ & $L_{1 / 4 / 7 / 10}^{a}$ & $L_{2 / 5 / 8 / 11}^{a}$ & $L_{3 / 6 / 9 / 12}^{a}$ & $L_{\mathrm{v}}$ & $L_{1 / 4 / 7 / 10}^{b}$ & $L_{2 / 5 / 8 / 11}^{b}$ & $L_{3 / 6 / 9 / 12}^{b}$ \\
\hline$m(\mathrm{~kg})$ & 31.930 & 2.250 & 2.36 & 32.75 & 0 & 1.880 & 2.360 & 34.690 \\
$a_{x}(\mathrm{~m})$ & - & 0.062 & 0 & 0 & 0 & 0 & -0.048 & 0 \\
$a_{y}(\mathrm{~m})$ & - & 0 & -0.003 & 0 & 0 & 0 & 0 & -0.010 \\
$a_{z}(\mathrm{~m})$ & - & 0 & 0 & -0.016 & 0 & 0.048 & 0 & 0 \\
$b_{x}(\mathrm{~m})$ & $0 /-0.243$ & 0.136 & 0 & 0 & 0 & 0 & -0.198 & 0 \\
$b_{y}(\mathrm{~m})$ & $0 / 0$ & 0 & -0.240 & 0 & 0 & 0 & 0 & -0.230 \\
$b_{z}(\mathrm{~m})$ & $0 / 0$ & 0 & 0 & 0.016 & 0 & -0.048 & 0 & 0 \\
$I_{x x}\left(\mathrm{~kg} \cdot \mathrm{m}^{2}\right)$ & 0.375 & 0.002 & 0.002 & 0.399 & 0 & 0.001 & 0.002 & 0.377 \\
$I_{\mathrm{yy}}\left(\mathrm{kg} \cdot \mathrm{m}^{2}\right)$ & 0.377 & 0.014 & 0.002 & 0.503 & 0 & 0.001 & 0.002 & 0.377 \\
$I_{z z}\left(\mathrm{~kg} \cdot \mathrm{m}^{2}\right)$ & 0.480 & 0.015 & 0 & 0.375 & 0 & 0 & 0.001 & 0.485 \\
\hline
\end{tabular}

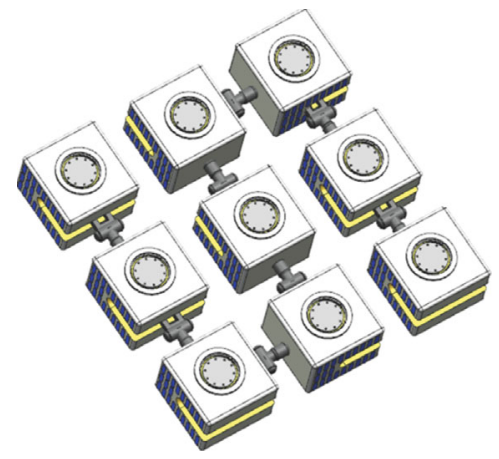

(a)

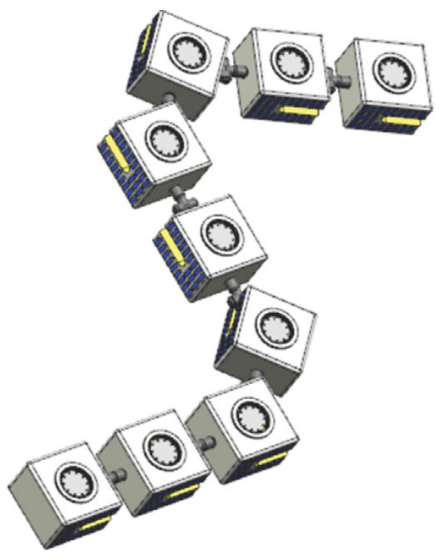

(b)

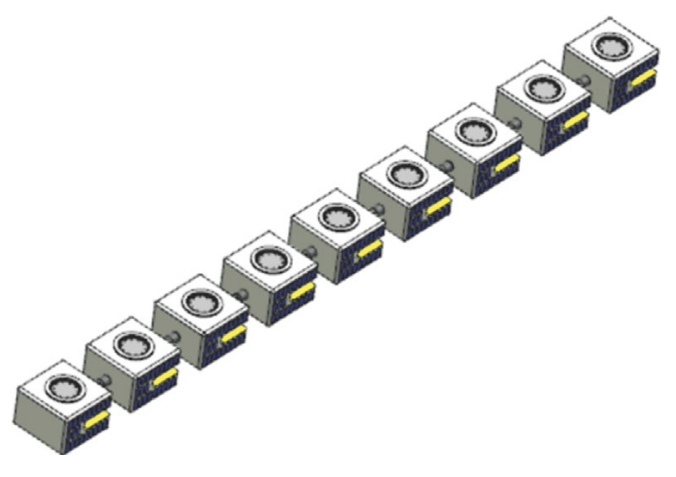

(c)

FIGURE 5: Process diagram of SMRS from the folded to unfolded state in case 1: (a) folded state; (b) unfolding state; (c) unfolded state.

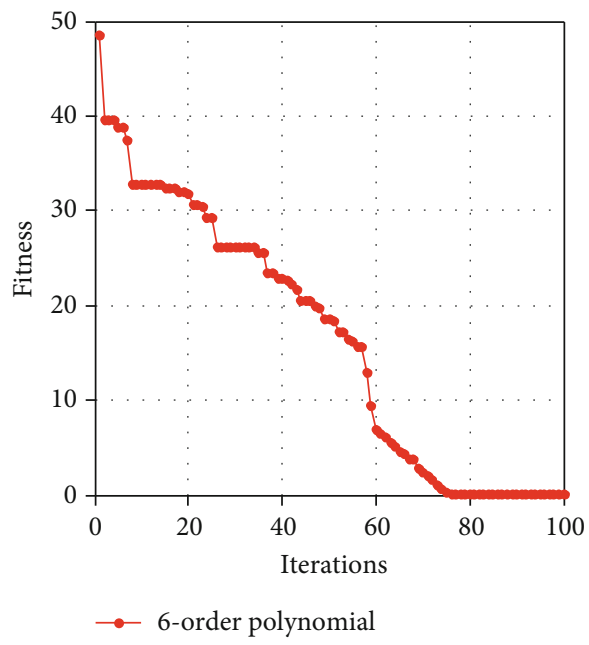

(a)

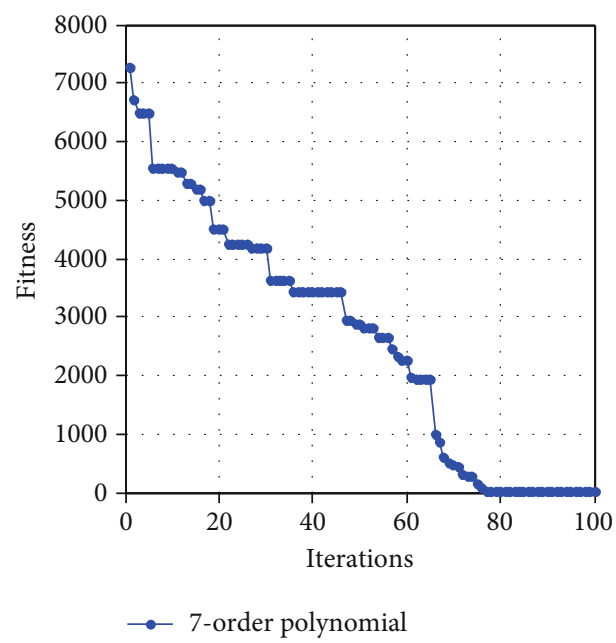

(b)

Figure 6: Fitness convergence curves of the PSO algorithm in case 1: (a) 6-order polynomial parameterized trajectories; (b) 7-order polynomial parameterized trajectories.

The optimization results in Figure 6(b) show that the optimal fitness value converges to 0.0484 based on the optimization vector which converges to $[0,0,0,0,0,0,0,0]$, and at this time, the highest and second highest order coeffi- cient of the 7-order polynomial are 0 ; then, the polynomial is converted to the 5-order polynomial, too.

The trajectory, velocity, and acceleration curves of joint trajectory parameterized by the 5-order polynomial are 


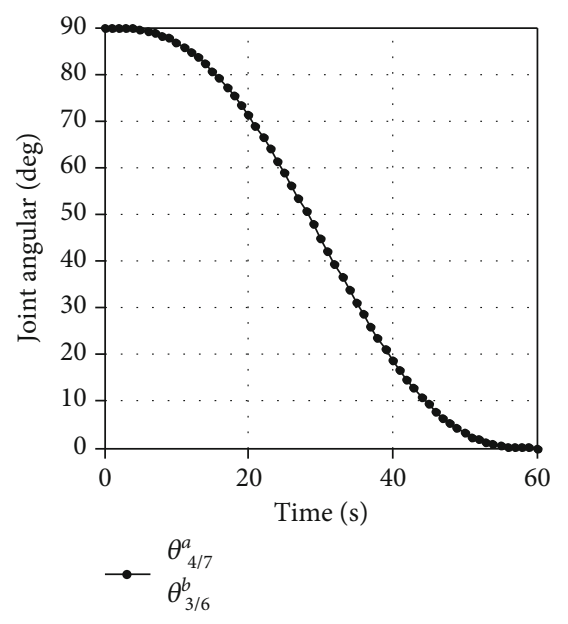

(a)

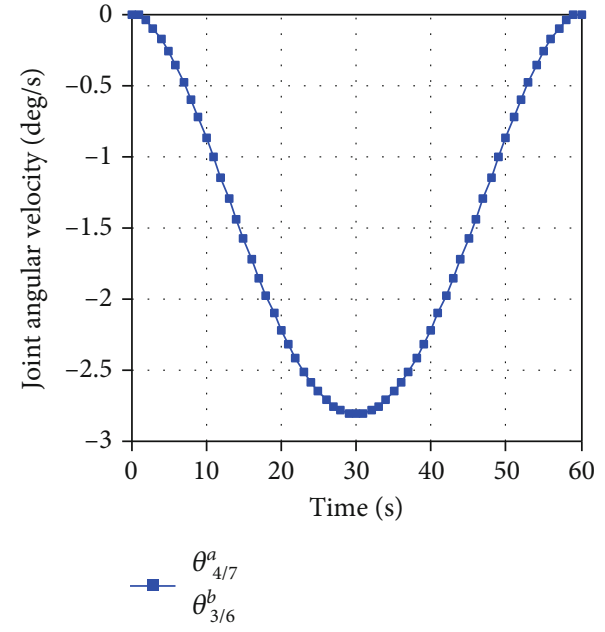

(b)

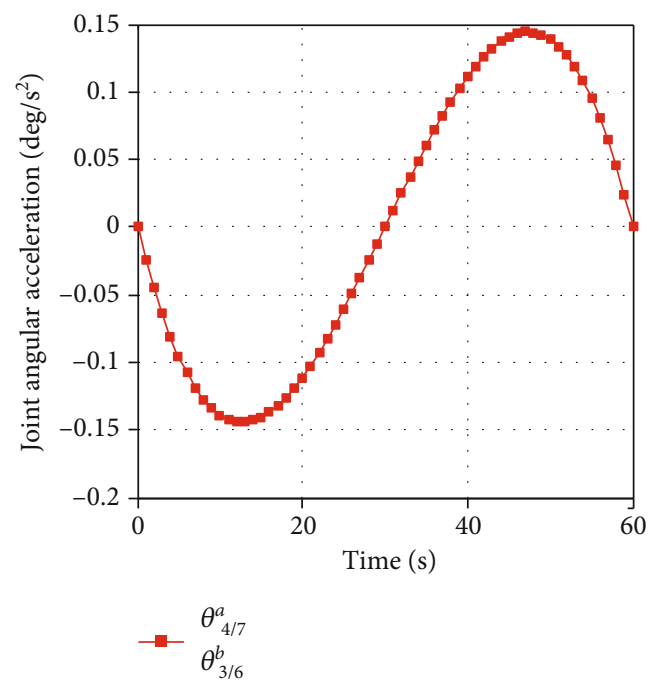

(c)

Figure 7: The joint angular trajectory, velocity, and acceleration curves of the 5-order polynomial in case 1: (a) the trajectory curves; (b) the velocity curves; (c) the acceleration curves.

shown in Figure 7, from which we can see that the trajectory of each joint is smooth and meets the requirements for the joint velocities and accelerations. The joint trajectory optimization results in the form of 6-order and 7-order polynomials prove that 5-order polynomials are ideal trajectory parameterization curves under the conditions of satisfying the velocities and accelerations of joint motions.

By introducing the joint trajectory in the form of 5-order polynomial into Equation (14), the pose velocity curves of $\Sigma_{b}$ from the folded state to unfolded state are obtained and shown in Figure 8 . We can see that the pose velocities of $\Sigma_{b}$ are small, and the reconfigurable process is stable because they are within the limited interval and have not changed drastically.

When the trajectory is parameterized by the 5-order polynomial, the curves related to pose velocities of $\Sigma_{b}$ and mission time are drawn in Figure 9. It indicates that with the prolongation of the mission time, the linear velocities and angular velocities of SMRS of $\Sigma_{b}$ slow down. When the mission time is fully prolonged, the satellite will reconfigure at very low velocities. When the maximum allowable angular velocity of $\Sigma_{b}$ is set to 0.005 , as shown in Figure 9, each velocity curve will have one intersection with the angular velocity threshold line; then, maximum abscissa of these intersections' read out is $400 \mathrm{~s}$, which means the minimum feasible mission time in case 1 is $400 \mathrm{~s}$.

5.2. Case 2. Case 2 conducts the trajectory planning simulation of SMRS from the unfolded state to a certain mission configuration, and their configurations are shown in Figures 10(a) and 10(b). The joint angle vector of SMRS in the unfolded state is $\Theta_{0}^{k}=[0,0,0,0,0,0,0,0,0,0,0,0],, k=a$ ,$b$. At the end of the mission, the state changes to a certain mission configuration whose joint angle vector is $\Theta_{f}^{k}=[0,0$ $\left., 0,0,10^{\circ}, 0,0,0,0,0,0,0\right], k=a, b$, and the nine modules of 


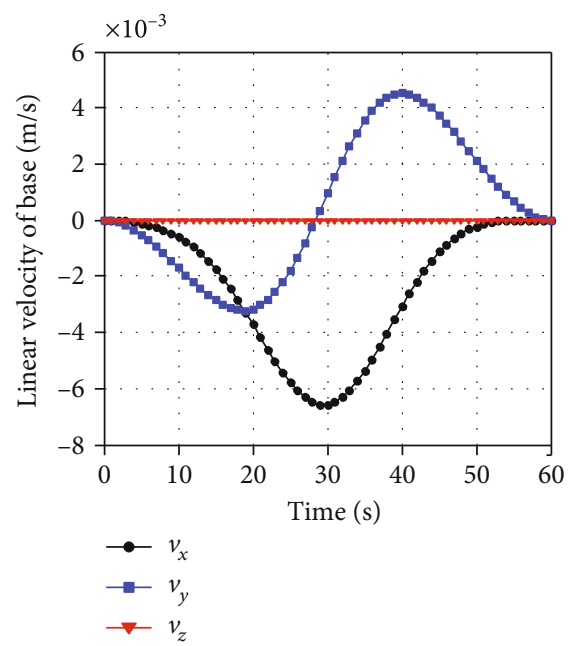

(a)

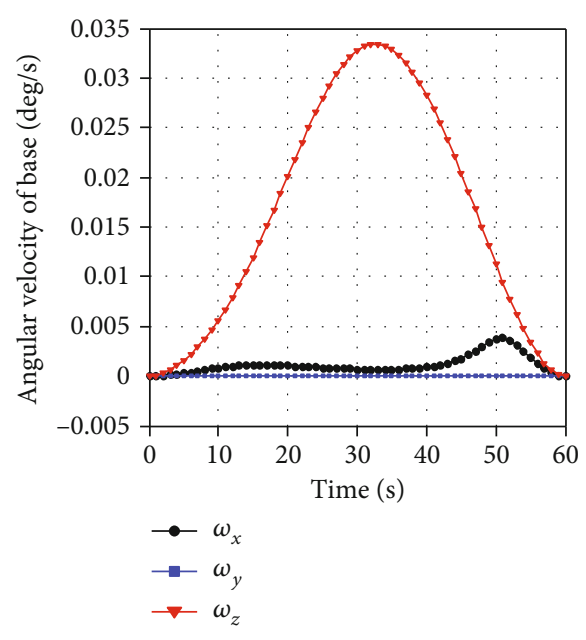

(b)

FIGURE 8: Linear velocities and angular velocities of the base coordinate system in case 1: (a) linear velocities; (b) angular velocities.

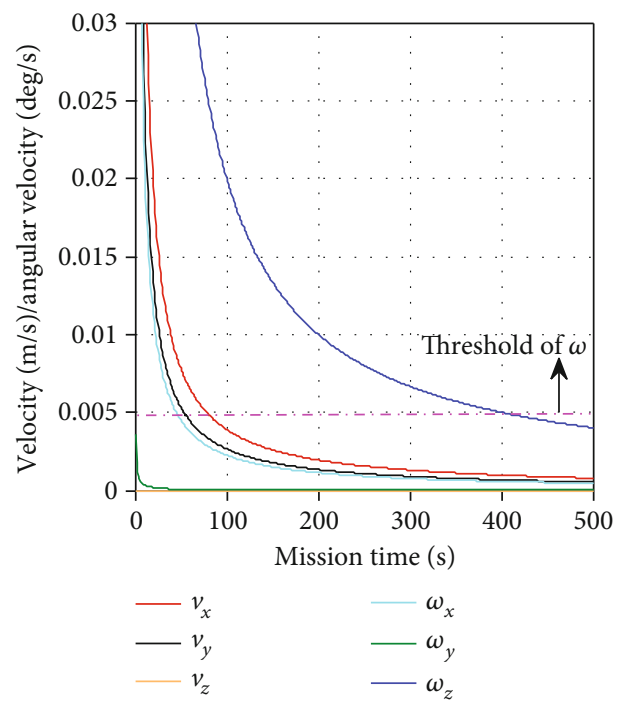

Figure 9: Curves related to pose velocities of the base coordinate system and mission time in case 1.

SMRS are divided into three groups; each group has three modules and has different directions relative to other groups. SMRS in this configuration can carry out reconnaissance missions in three directions simultaneously. The initial time is set to 0 and mission time to $60 \mathrm{~s}$. In order to ensure the smoothness of joint trajectories, the joint angular velocities and accelerations at initial time are set to 0 .

The parameter setting of the particle swarm algorithm in case 2 is consistent with that in case 1 . While planning joint trajectories in the form of 6-order polynomials in case 2 , the optimization parameter vector composed of 2 optimization parameters is $\left[a_{56}^{a}, a_{56}^{b}\right]$; while planning joint trajectories in the form of 7-order polynomials, the optimization parameter vector composed of 4 optimization parameters is $\left[a_{56}^{a}\right.$, $\left.a_{56}^{b}, a_{57}^{a}, a_{57}^{b}\right]$.
The PSO algorithm is used to optimize the trajectory parameters in the form of 6-order polynomial and 7-order polynomial, respectively, and their convergence process of fitness is shown in Figures 11(a) and 11(b).

In Figure 11(a), the fitness value converges to 0.02 while the optimization vector converges to $[0,0]$, and at this time, the highest order coefficient of the 6-order polynomial is 0 ; then, the polynomial is converted to the 5-order polynomial.

In Figure 11(b), the fitness value converges to 0.02 based while the optimization vector converges to $[0,0,0,0]$, and at this time, the highest and second highest order coefficient of the 7 -order polynomial are 0 ; then, the polynomial is converted to the 5-order polynomial, too.

In Figure 11, the optimization results of the trajectory in case 2 in the form of 6-order and 7-order polynomials prove that the 5-order polynomials are ideal trajectory parameterized curves under the conditions of satisfying the velocity and acceleration of joint motions. The trajectory, velocity, and acceleration curves of joint trajectories parameterized by 5-order polynomial are shown in Figure 12; we can see that the joint trajectory is smooth and meets the requirements of joint trajectory velocity and acceleration.

The joint trajectory in the form of 5-order polynomial is introduced into Equation (14), and the velocity curves of three position directions and three joint axis angle changes of $\Sigma_{b}$ in the process of reconfiguration from the unfolded state to mission state are obtained. What we can see from Figure 13 is that the pose velocities of the base coordinate system are within the limited interval and have not changed drastically, and the reconfigurable process is relatively stable.

When the trajectory is parameterized by the 5-order polynomial, the curves related to pose velocities of $\Sigma_{b}$ and mission time are drawn in Figure 14. It indicates that with the prolongation of mission time, the linear velocities and angular velocities of SMRS of $\Sigma_{b}$ slow down. When the mission time is fully prolonged, the satellite will change its pose 


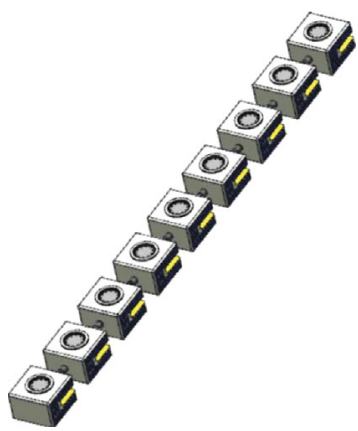

(a)

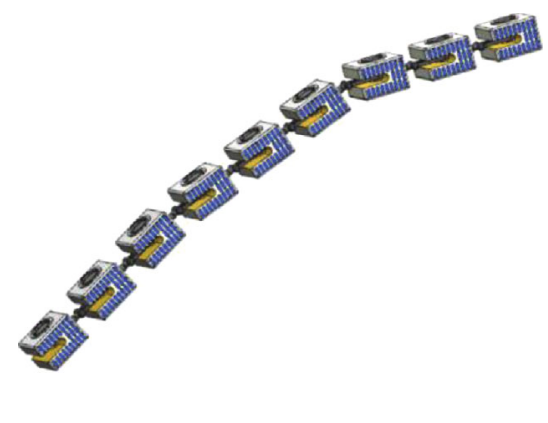

(b)

FIgure 10: Process of SMRS from the unfolded state to mission state in case 2: (a) unfolded state; (b) mission state.

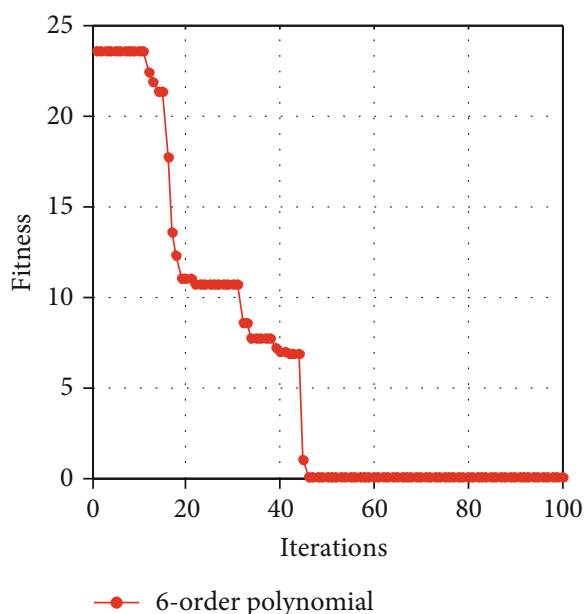

(a)

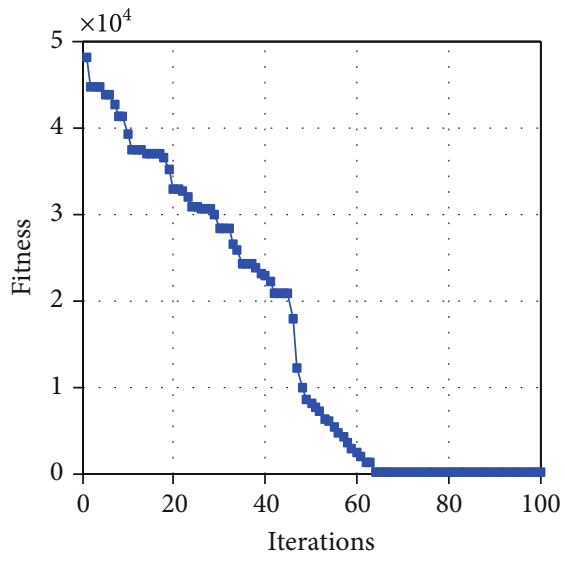

$\rightarrow$ 7-order polynomial

(b)

FIGURE 11: Fitness convergence curves of the PSO algorithm in case 2: (a) 6-order polynomial parameterized trajectories; (b) 7-order polynomial parameterized trajectories.

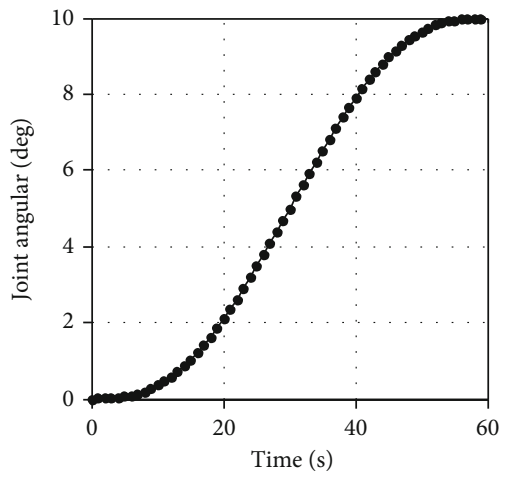

$-\theta_{5}^{a}$

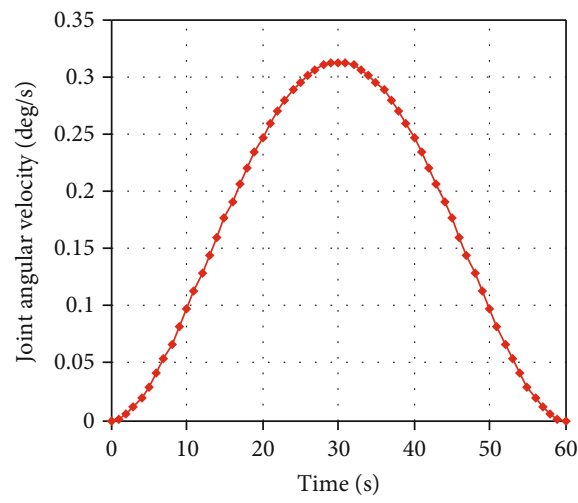

$-\begin{aligned} \theta_{5}^{a} \\ \theta_{5}^{b}\end{aligned}$

(a)

(b)

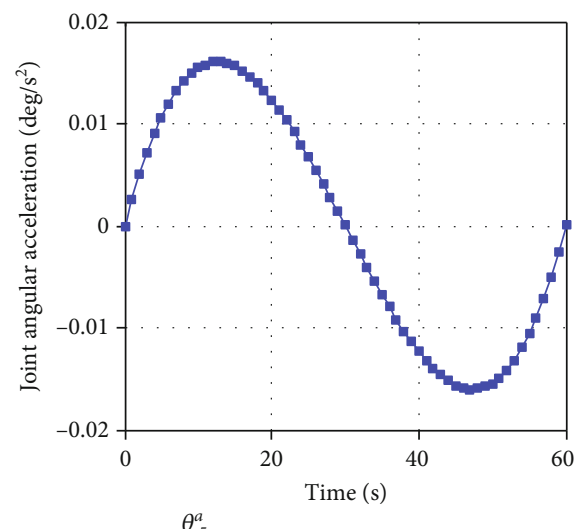

$-\begin{array}{r}\theta_{5}^{a} \\ \theta_{5}^{b}\end{array}$

(c)

FIgURE 12: The angular trajectory, velocity, and acceleration curves of joint angles of 5-order polynomial in case 2: (a) the trajectory curves; (b) the velocity curves; (c) the acceleration curves. 


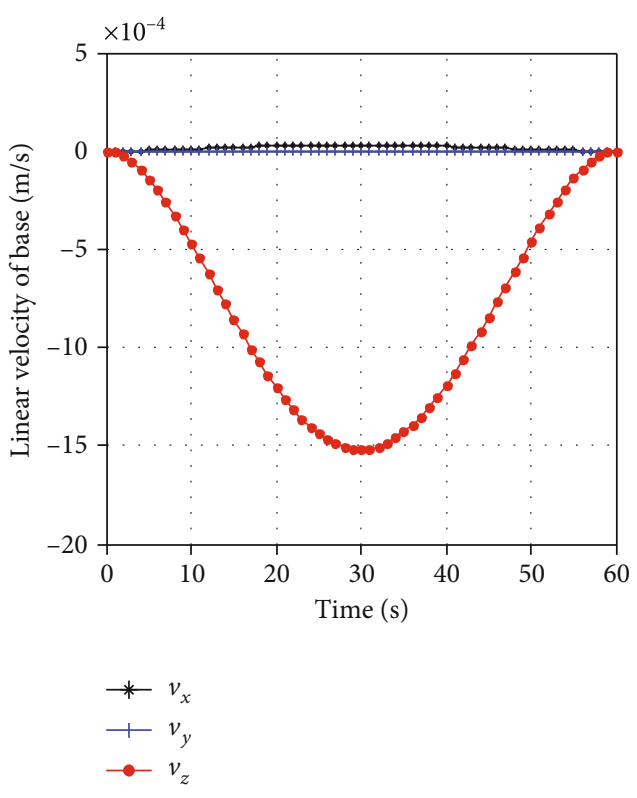

(a)

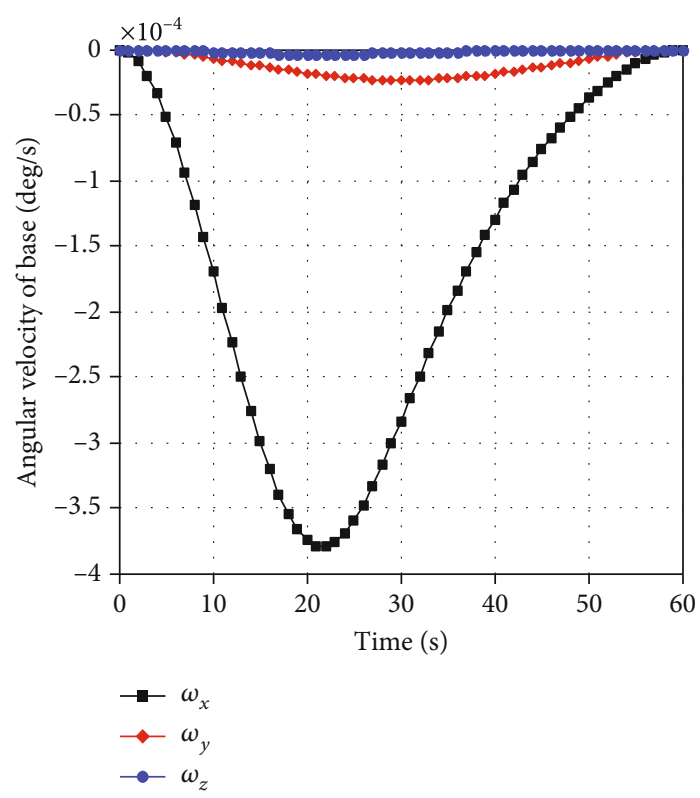

(b)

Figure 13: Linear velocities and angular velocities of the base coordinate system in case 2: (a) linear velocities; (b) angular velocities.

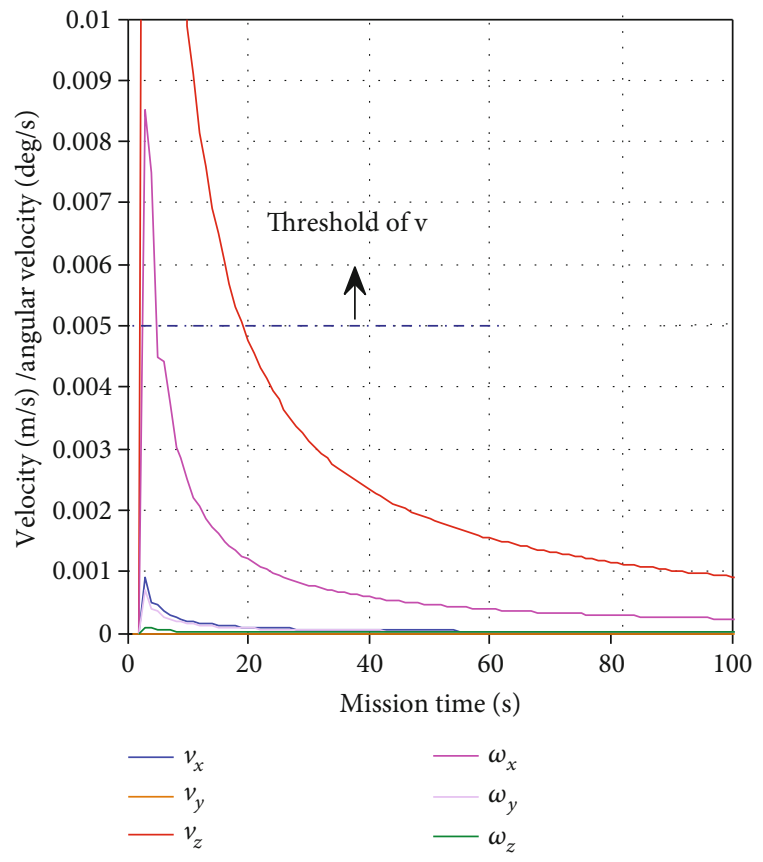

Figure 14: Curves related to pose velocities of the base coordinate system and the mission time in case 2 .

at small velocities. According to Figure 14, the minimum mission time with threshold of pose velocity can be read. When the maximum allowable linear velocity of $\Sigma_{b}$ is set to 0.005, as shown in Figure 14, each velocity curve will have one intersection with the linear velocity threshold line; then, the maximum abscissa of these intersections' read out is $20 \mathrm{~s}$, which means the minimum feasible mission time in case 2 is $20 \mathrm{~s}$.

\section{Conclusion}

Based on the reality that the traditional space system can only perform a single mission and is difficult to respond quickly to emergencies, this paper proposes a new concept spacecraft named space modular reconfigurable satellites which is expected to perform multiple space missions and respond to unforeseen events by carrying multiple mission subsystems and structural reconfiguration. In the process of adaptive reconfiguration of SMRS, there is a coupling effect between the motion of each joint and the pose of each modules. Aiming at the stability of the reconfiguration process, the paper established the kinematic equations of SMRS, and the joint trajectories of SMRS are parameterized by the 6order and 7-order polynomials, respectively. The trajectories of SMRS from folded configuration to unfolded configuration and from unfolded configuration to a mission configuration are planned by the PSO algorithm. The main conclusions are as follows:

(1) In the process of adaptive reconfiguration of SMRS, there is a coupling effect between the joints motion and the pose of each module which provides theoretical support for improving the reconfiguration stability of SMRS by joint trajectory plan

(2) The D-H method based on the virtual joint coordinate system can effectively solve the problem of space orientation asymmetry of reconfigurable joints axis on both sides of base coordinate system of SMRS

(3) The PSO algorithm for trajectory planning can quickly converge, and the optimized trajectories meet the set constraints. The consistent results of different parameterized curves in one case prove the PSO 
algorithm is an ideal intelligent algorithm for trajectory planning of SMRS

This paper completed research on the kinematic modeling and trajectory planning of SMRS. The research results provide a reference for the kinematics and dynamics modeling, control, and joint trajectory selection of SMRS and other reconfigurable space systems.

\section{Data Availability}

The data used to support the findings of this study are included within the article.

\section{Conflicts of Interest}

The authors declare no conflict of interest.

\section{Acknowledgments}

This research was funded by National Defense Science and Technology Innovation Zone of China, grant number 00205501.

\section{References}

[1] Y. Zhang, W. Wang, J. Sun, H. Chang, and P. Huang, "A selfreconfiguration planning strategy for cellular satellites," IEEE Access, vol. 7, pp. 4516-4528, 2019.

[2] M. Nisser, D. Izzo, and A. Borggraefe, "An electromagnetically actuated, self-reconfigurable space structure," Transactions of the Japan Society for aeronautical and space sciences, vol. 14, pp. 1-9, 2017.

[3] C. Kief, M. Hannon, J. Lyke, C. Peters, D. Fronterhouse, and M. Ahlberg, "SPARC - 1: a new, improved modular 6U spacecraft," in 2019 IEEE Aerospace Conference, Big Sky, MT, USA, March 2019.

[4] X. Huang, Y. Yan, and Y. Zhou, "Underactuated spacecraft formation reconfiguration with collision avoidance," Acta Astronautica, vol. 131, pp. 166-181, 2017.

[5] M. Kortman, S. Ruhl, J. Weise et al., "Building block based iBoss approach: fully modular systems with standard interface to enhance future satellites," in 66th International Astronautical Congress (Jerusalem), 2015Jerusalem, Israel.

[6] C. Underwood, S. Pellegrino, V. Lappas et al., Autonomous assembly of a reconfiguarble space telescope (AAReST)-a CubeSat/microsatellite based technology demonstrator27th Annual AIAA/USU Conference on Small Satellites, Logan, Utah, 2013.

[7] F. Matsumoto and H. Yoshimura, "Dynamics and trajectory planning of a space robot with control of the base attitude," in IUTAM Symposium on Dynamics Modeling and Interaction Control in Virtual and Real Environments, Springer Netherlands, Dordrecht, 2011.

[8] L. Yan, W. Xu, Z. H. Hu, and B. Liang, "Virtual-base modeling and coordinated control of a dual-arm space robot for target capturing and manipulation," Multibody System Dynamics, vol. 45, no. 4, pp. 431-455, 2019.

[9] X. P. Wei, J. X. Zhang, D. S. Zhou, and Q. Zhang, "Optimal path planning for minimizing base disturbance of space robot," International Journal of Advanced Robotic Systems, vol. 13, no. 2, p. 41, 2017.
[10] X. Zhao, Z. Xie, H. Yang, and J. Liu, "Minimum base disturbance control of free-floating space robot during visual servoing pre-capturing process," Robotica, vol. 38 , no. 4, pp. $652-$ 668,2020

[11] E. G. Kaigom, T. J. Jung, and J. Roßmann, "Optimal motion planning of a space robot with base disturbance minimization," in 11th Symposium on Advanced Space Technologies in Robotics and Automation, 2011Noordwijk, Netherlands.

[12] X. Liu, C. Qiu, Q. Zeng, and A. Li, "Kinematics analysis and trajectory planning of collaborative welding robot with multiple manipulators," Procedia CIRP, vol. 81, pp. 1034-1039, 2019.

[13] V. V. M. J. S. Chembuly and H. K. Voruganti, "Trajectory planning of redundant manipulators moving along constrained path and avoiding obstacles," Procedia Computer Science, vol. 133, pp. 627-634, 2018.

[14] L. Wang, Q. Wu, F. Lin, S. Li, and D. Chen, "A new trajectoryplanning beetle swarm optimization algorithm for trajectory planning of robot manipulators," IEEE Access, vol. 7, pp. 154331-154345, 2019.

[15] H. I. Lin, "A fast and unified method to find a minimum-jerk robot joint trajectory using particle swarm optimization," Journal of Intelligent \& Robotic Systems, vol. 75, no. 3-4, pp. 379-392, 2014.

[16] J. Luo, L. Zong, M. Wang, and J. Yuan, "Optimal capture occasion determination and trajectory generation for space robots grasping tumbling objects," Acta Astronautica, vol. 136, pp. 380-386, 2017.

[17] W. Xu, Y. Liu, and Y. Xu, "The coordinated motion planning of a dual-arm space robot for target capturing," Robotica, vol. 30, no. 5, pp. 755-771, 2012.

[18] K. Mohamed, H. Elgamal, and A. Elsharkawy, "Dynamic analysis with optimum trajectory planning of multiple degree-offreedom surgical micro-robot," Alexandria Engineering Journal, vol. 57, no. 4, pp. 4103-4112, 2018.

[19] H. Liu, X. Lai, and W. Wu, "Time-optimal and jerkcontinuous trajectory planning for robot manipulators with kinematic constraints," Robotics and Computer-Integrated Manufacturing, vol. 29, no. 2, pp. 309-317, 2013.

[20] L. Yan, W. Xu, Z. Hu, and B. Liang, "Multi-objective configuration optimization for coordinated capture of dual-arm space robot," Acta Astronautica, vol. 167, pp. 189-200, 2020.

[21] "Optimal trajectory planning for spherical robot using evolutionary algorithms," Procedia Manufacturing, vol. 32, pp. 960-968, 2019.

[22] H. Wang, H. Wang, J. Huang, B. Zhao, and L. Quan, "Smooth point-to-point trajectory planning for industrial robots with kinematical constraints based on high-order polynomial curve," Mechanism and Machine Theory, vol. 139, pp. 284-293, 2019.

[23] M. Wang, J. Luo, J. Yuan, and U. Walter, "Coordinated trajectory planning of dual-arm space robot using constrained particle swarm optimization," Acta Astronautica, vol. 146, pp. 259-272, 2018.

[24] X. Liu, C. Qiu, Q. Zeng, A. Li, and N. Xie, “Time-energy optimal trajectory planning for collaborative welding robot with multiple manipulators," Procedia Manufacturing, vol. 43, pp. 527-534, 2020.

[25] Y. Li, T. Huang, and D. G. Chetwynd, "An approach for smooth trajectory planning of high-speed pick-and-place parallel robots using quintic B-splines," Mechanism and Machine Theory, vol. 126, pp. 479-490, 2018. 
[26] C. Qin and X. Gu, "Improved PSO algorithm based on exponential center symmetric inertia weight function and its application in infrared image enhancement," Symmetry, vol. 12, no. 2, p. 248, 2020.

[27] J. Lan, Y. Xie, G. Liu, and M. Cao, “A multi-objective trajectory planning method for collaborative robot," Electronics, vol. 9, no. 5 , p. $859,2020$.

[28] S. Kucuk, "Maximal dexterous trajectory generation and cubic spline optimization for fully planar parallel manipulators," Computers \& Electrical Engineering, vol. 56, pp. 634-647, 2016.

[29] Y. Du and F. Xu, "A hybrid multi-step probability selection particle swarm optimization with dynamic chaotic inertial weight and acceleration coefficients for numerical function optimization," Symmetry, vol. 12, no. 6, p. 922, 2020. 